\title{
Sixième compte rendu du séminaire sur les Pensées de Pascal
}

Dominique Descotes

\section{(2) OpenEdition \\ 12 Journals}

Édition électronique

URL : http://journals.openedition.org/ccibp/487

DOI : $10.4000 /$ ccibp. 487

ISSN : 2493-7460

Éditeur

Centre international Blaise Pascal

\section{Édition imprimée}

Date de publication : 15 décembre 2008

Pagination : 15-40

ISBN : 978-2-84516-406-2

ISSN : 0249-6674

\section{Référence électronique}

Dominique Descotes, «Sixième compte rendu du séminaire sur les Pensées de Pascal », Courrier du Centre international Blaise Pascal [En ligne], 30 | 2008, mis en ligne le 30 novembre 2015, consulté le 14 septembre 2020. URL : http://journals.openedition.org/ccibp/487

Ce document a été généré automatiquement le 14 septembre 2020

Centre international Blaise Pascal 


\section{Sixième compte rendu du séminaire sur les Pensées de Pascal}

\section{Dominique Descotes}

\section{Laf. 59, Sel. 93}

Quand il est question de juger si on doit faire la guerre et tuer tant d'hommes, condamner tant d'Espagnols à la mort, c'est un homme seul qui en juge, et encore intéressé : ce devrait être un tiers indifférent.

RO 67-5

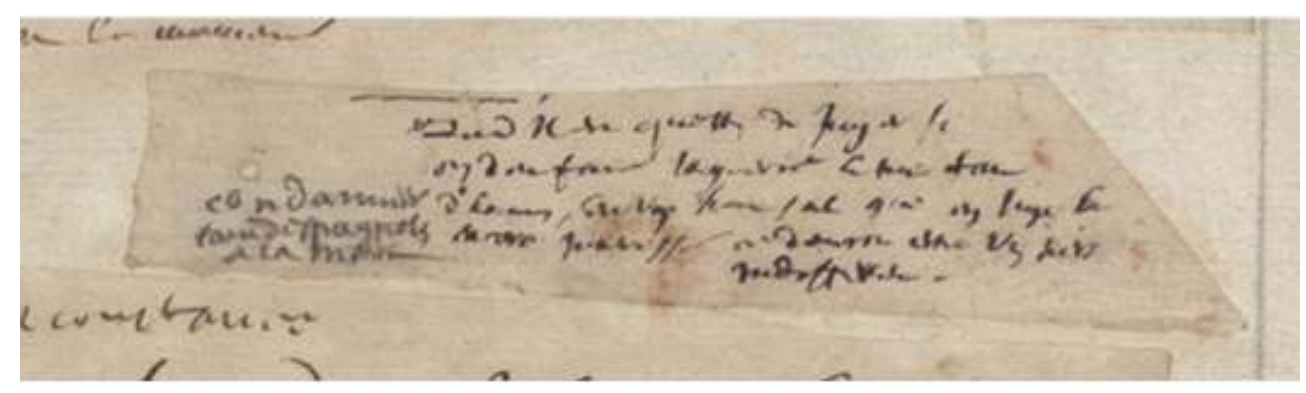

Quand il est question de juger si

on doit faire la guerre \& tuer tant

condamner d'hommes, cest un homme seul qui en juge \&

tant despagnols encore interessé ce devroit estre un tiers

a la mort indifferent.

1 D’après Y. Maeda, le texte est un autographe de Pascal, et tous les éditeurs pensent de même. Noter toutefois que l'écriture de ce fragment est la même que celle de Laf. 49, Sel. 82. Pour Laf. 49, Sel. 82, que certains éditeurs (Michaud, Brunschvicg minor, Lafuma Luxembourg) ont considéré comme un fragment non autographe de Pascal.

2 Le trou d'enfilage est visible à gauche. 

est certaine, en raison des marques textuelles communes des papiers :

- Laf. 531 (de XXIII), Sel. 456.

- Laf. 49, Sel. 82, de Vanité.

- Laf. 59, Sel. 93, de Misère.

4 Tourneur signale déjà la succession Laf. 49, Sel. 82 et Laf. 59, Sel. 93. D'après Tourneur, Pascal a d'abord écrit le texte de ce fragment sur une feuille au-dessous du fragment Laf. 49, Sel. 82. Ensuite il aurait découpé les deux fragments et classé Laf. 59, Sel. 93 dans Misère et Laf. 49, Sel. 82 dans Vanité.

5 Condamner tant d'Espagnols à la mort, d'après Tourneur, est une addition au crayon repassée à l'encre. Les éditions intercalent condamner tant d'Espagnols à la mort entre tuer tant d'hommes et c'est un homme. Mais il n'y a pas de renvoi dans le texte pour condamner tant d'Espagnols à la mort. Si on supprime l'addition, le fragment se relie facilement à César.

\section{Interprétation du fragment}

10 L'idée de guerre relie ce fragment avec le précédent, surtout quand on considère que les Espagnols sont absents de la première rédaction. Il s'agit de la question du pouvoir décisionnaire. aboutirent en 1659 au traité des Pyrénées. Pascal reprocherait au roi d'Espagne de s'être longtemps refusé à la paix et d'avoir fait verser pour son ambition le sang de ses sujets à la bataille des Dunes (14 juin 1658). L'idée est reprise par Maeda et Le Guern. Havet, Tourneur, Anzieu, Lafuma (Luxembourg) font aussi référence à la bataille des Dunes ; mais Maeda ajoute que ce n'est qu'une hypothèse. En s'appuyant sur la date de cette bataille, Lafuma tente de fixer la date de la conférence de Pascal à Port-Royal ; voir Controverses pascaliennes, p. 43 ; Maeda n'est pas d'accord.

15 La Provinciale XIV, § 21-24, contient un long développement sur les conditions qui permettent de tuer légitimement des hommes.

\section{Laf. 60, Sel. 94 - Laf. 76, Sel. 111}

Voir la reconstitution de la genèse de ce texte dans MAEDA Yoichi, Commentaires, III, p. 4 sq.

Courrier du Centre international Blaise Pascal, 30 | 2008 


\section{Structure d'ensemble des folios 69-70 et 365-366} p. 293. Papier au filigrane raisin. Voir p. 280 : papiers appartenant à un paquet de 5 feuilles (soit 10 feuillets), dont chaque feuillet est marqué d'un filigrane identique, une grappe de raisin. rectos et les versos desquels Pascal a écrit les textes désignés chez Sellier par les numéros 94 et 111.

\section{Verso de la première page, folio $n^{\circ} 70$}

\section{Verso de la première page, folio $\mathrm{n}^{\circ} 70$}

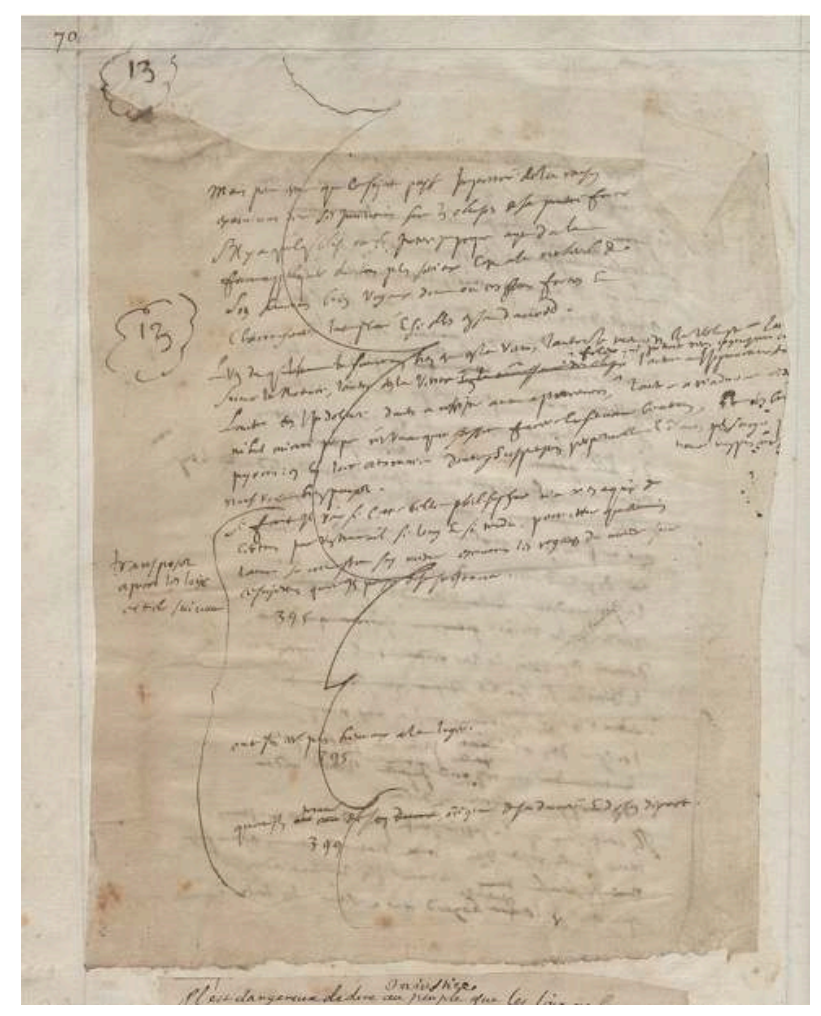

Cliché BNF

Sur cette page du Recueil original, on voit que le papier a été collé, mais qu'il restait un peu de place en bas, ce qui a permis de coller en-dessous un autre fragment, qui n'a rien à voir avec lui.

Dans l'indication en marge à gauche, Brunschvicg lit au titre suivant. À partir de Tourneur, tous les éditeurs sont d'accord sur article suivant. 
Sur le sens du mot Article, voir le Petit traité des solides circulaires, composé de 9 articles, qui se suivent sans être solidement liés les uns aux autres, alors que les autres traités contiennent des Propositions (ou des Propriétés, dans le cas particulier du Propriétés des sommes) dont l'enchaînement logique est rigoureux. De même pour le Traité général de la roulette. Cela semble insister sur le caractère disjoint des éléments, quoique appartenant tous à un même ensemble.

Sur les parties barrées de ce texte, voir dans $O C$ III, p. 102 sq., l'analyse de J. Mesnard sur les recueils d'excerpta constitués par Pascal. Le texte du fragment Laf. 60-76, Sel. 94-111 a été rayé dans le manuscrit original : s'il a tout de même été conservé, c'est parce que le verso du feuillet qui le portait a servi pour la rédaction d'un autre fragment. Il était devenu sans objet, comme dans la plupart des cas semblables, parce que Pascal en avait repris la substance dans d'autres textes. Quoique Montaigne ne soit nommé à aucun moment dans ce fragment des Pensées, c'est lui qui inspire tout le développement, comme en témoignent les références aux pages de l'édition de 1652 des Essais. Il s'agit d'un brouillon dans lequel les passages de Montaigne sont parfois intégrés à une expression propre à Pascal, et parfois réduits à de simples références. Il ne s'agit pas alors de présenter Montaigne comme c'est le cas dans l'Entretien avec M. de Sacy, mais d'exploiter son œuvre comme un répertoire de faits et d'arguments.

Cette différence de perspective peut permettre de situer chronologiquement l'Entretien par rapport aux Pensées. Les excerpta de Montaigne mis en œuvre se fondent parfois, au début par exemple, dans le texte de Pascal. Ils ne signifient pas pour autant que ces textes des Pensées sont antérieurs à l'Entretien. Il ne s'agit pas de la même perspective, comme l'indique J.Mesnard. Il est donc possible que les deux textes soient complètement disjoints.

Ces remarques permettent d'expliquer le peu de rapport de ces notes avec le texte complètement rédigé.

8 Voir ce qu'indique Tourneur dans son édition, p. 184-185. Ces textes sont écrits sur les deux faces de deux feuillets. Le premier de ces feuillets, sans doute " côté A » (Folio 69), dont le haut a été retranché, contenait sur une face le début du texte sur les lois, et sur l'autre face (appelée par Tourneur " côté B ", qui correspond au folio 70) le texte qui commence par Mais peut-être..., jusqu'au nombre 399. Ce texte porte, dans la marge de gauche, le nombre 12, corrigé en 13, dans un cartouche. Plus bas, Pascal a tracé une accolade qui embrasse la partie du texte qui commence par Si faut-il et mis en marge transposez après les lois article suivant, puis barré la page dans tout sa longueur par un trait en zigzag. Enfin le feuillet a été collé sur la page 69 du recueil, qui a été crevée pour laisser voir, au verso, le texte barré, et une main étrangère a repris sur le haut du cadre qui les cachait, le chiffre 13 dans un cartouche et le trait en zigzag. Quant au second feuillet, qui portait sur la première face, côté B, et en haut du verso, la fin du texte sur les lois, suivie du texte qui commence par Est-ce dont que l'âme, et que Pascal avait coté du nombre 12, entouré d'un cartouche et corrigé en 13 et qui est barré d'un trait vertical, il a été collé sur la p. 365 du recueil, qui a été crevée pour laisser voir le verso.

En fait, Tourneur semble faire une erreur : c'est sur le folio 70 que le papier est collé. C'est le 69 que l'on voit à travers une fenêtre. 


\section{accolades, mais des signes de
en trouve des exemples dans}

- Laf. 634, Sel. 527.

- Laf. 269 (qui n'effectue pas la transposition), Sel. 30 (qui transpose, comme Tourneur).

- Laf. 58, Sel. 91, Tyrannie.

- Laf. 432 (qui ne transpose pas, de même que Faugère, Brunschvicg, et Tourneur), Sel. 662.

- Laf. 957, Sel. 792, avec une accolade à droite.

- Laf. 877, Sellier 441.

- Laf. 969, Sel. 801, qui transposent, contrairement à Faugère, Brunschvicg et Le Guern.

Dans le cas du folio 70, le trait d'accolade est prolongé sur les pages suivantes, de telle manière qu'on peut le voir sur le papier. Sur l'ensemble du fragment, toute la partie non barrée est marquée d'un long trait à gauche. Pascal entendait donc transposer les éléments qui sont définis par l'accolade après la partie non barrée.

Le renvoi en marge indique qu'il faut transposer après les lois, avec la mention Article suivant.

Première page, Folio $n^{\circ} 69$ 


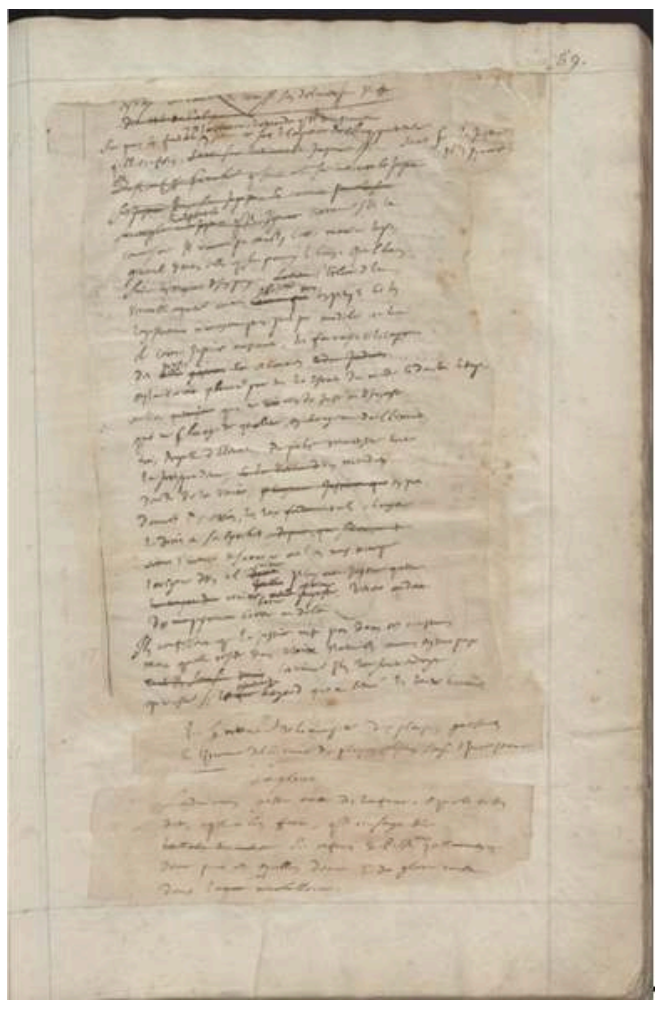

Cliché BNF

41 Les Folios 69 et 70 sont des recto-verso. Le grand texte du folio 69, qui forme le début de Laf. 60, Sel.94, est visible par une fenêtre découpée dans le papier du recueil ; ce qui signifie que le papier du manuscrit a été collé sur l'autre face de la feuille.

Ce papier était, avant le découpage, une page de gauche. Il ne constitue pas un feuillet entier. Il a été coupé en haut, de sorte qu'une partie du texte barrée est perdue. Il ne reste que ce qui commence par En vérité...

Les premières lignes du folio 69 sont barrées. Pourquoi Pascal les a-t-il barrées ? M. Le Guern pense que cette partie barrée devait avoir un rapport avec ce qui précédait, et qui est perdu par le découpage. Il pense que c'est la matière de ce qui se trouve au verso, et considère donc que les éléments qui se trouvent dans Laf. 76, Sel. 111 doivent être placés partie avant, partie après le texte de Laf. 60, Sel. 94.

Le découpage a conservé la partie en haut à droite pour ne pas perdre la formule Sera-ce sur la justice? Il l'ignore.

Le folio 365 porte une lettre B majuscule en haut à droite. Peut-être que le A est caché en haut à droite de ce papier, dissimulé par le cache, à hauteur de la partie barrée. Comme la fenêtre laissait un peu de place vers le bas, deux autres papiers ont pu être collés en-dessous.

Suivant Y. Maeda, le texte comporte un grand nombre de corrections du premier jet. Tourneur et Brunschvicg ne donnent pas le même texte barré. Dans le premier jet, Pascal ne fait d'abord allusion qu'à des éléments relevant du registre local. Ce n'est que plus tard qu'il se décide à introduire des éléments relevant du registre temporel. 
Recto de la deuxième feuille, folio $n^{\circ} 365$

Pour les folios 365 et 366, c'est la partie 365 qui est collée, et la partie 366 qui est visible à partir d'une fenêtre.

Folio 365

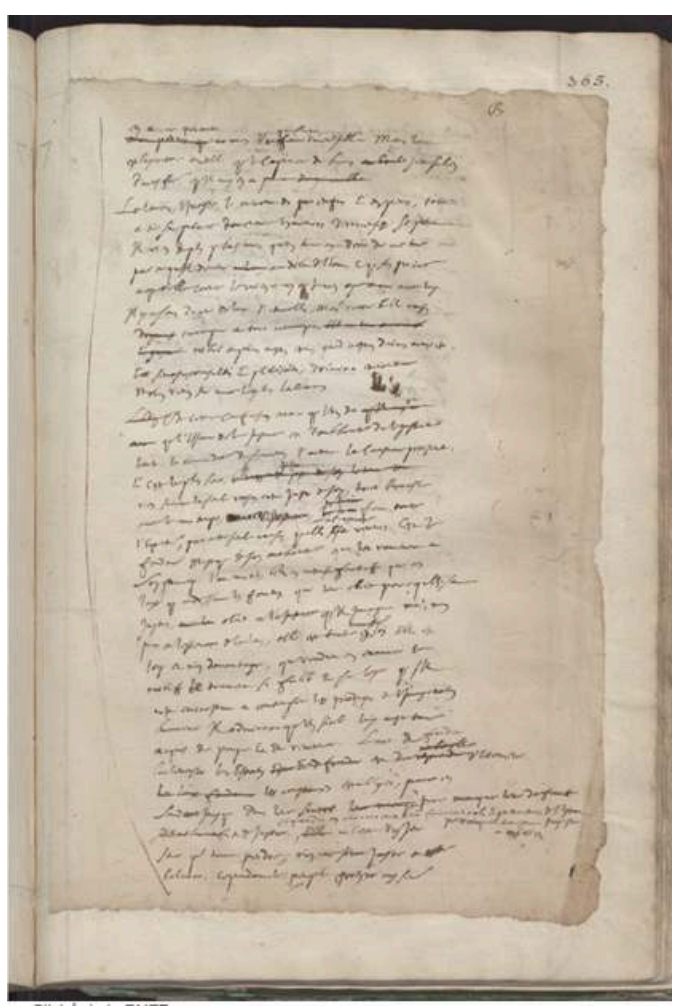

Cliché BNF

Page 366, verso 


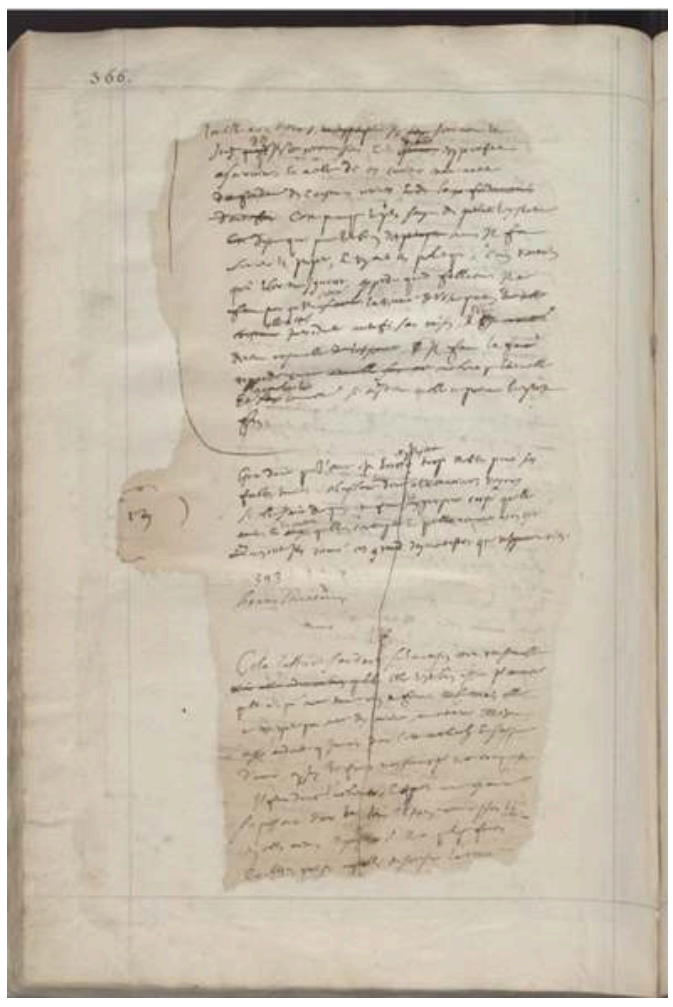

Page de droite, intérieur

Cliché BNF

GHEERAERT Tony, À la recherche du Dieu caché. Introduction aux Pensées de Pascal, La Bibliothèque électronique de Port-Royal, 2007, p. 148 sq. Commentaire général du fragment.

\section{Folio 69}

\section{[En vérité la vanité des lois il s'en délivrerait, il est donc utile de l'abuser.]}

51 Une partie du texte est barrée en haut du papier.

Elle semble se lire : En vérité la vanité des lois il s'en délivrerait, il est donc utile de l'abuser. Il manque apparemment, avant cette phrase, une partie barrée d'environ ou 6 lignes, selon Tourneur, peut-être plus. Il y a donc eu un texte embrayeur, que Pascal a supprimé : apparemment, ce serait un fragment proche de Sel. 27. Le début coïncide avec la conclusion du texte sur les lois : expedit quod fallatur. Pascal a transformé la suite en début de son grand texte. C'est une démarche qui est proche de la démarche du mathématicien, qui énonce d'abord sa proposition, puis en donne la démonstration. Mais dans un texte non technique, il est naturel, ensuite, de supprimer la conclusion formulée d'avance.

Le Guern pense que la partie barrée perdue par le découpage avait pour matière ce qui se trouve au verso, et considère donc que les éléments qui se trouvent dans Laf. 76, Sel. 111 doivent être placés partie avant, partie après le texte de Laf. 60, Sel. 94.

En revanche, l'édition Sellier, qui suit la Copie, rejette toutes les parties barrées dans Laf. 76, Sel. 111. 
Sur quoi fondera-t-il l'économie du monde qu'il veut gouverner? Sera-ce sur le caprice de chaque particulier? Quelle confusion! sera-ce sur la justice ? il l'ignore.

Le texte initial restitué par Maeda est :

Sur quoi les fondera on sera ce sur le caprice de chaque particulier quelle confusion, sera ce sur la véritable justice, Qu'il confesse franchement que si ce n'est sur la véritable justice ses justice lois sert injustes, Et comment peut la scie se régler à la justice qu'il ignore.

Pascal commence par écrire "Sur quoi les (ou la) fondera-t-on... " D’après Faugère, Tourneur et Maeda le mot les est barré. Les deux Copies, Brunschvicg, Chevalier, Strowski, Lafuma, Sellier donnent la non barré : Sur quoi la fondera-t-il l'économie... Il est rajouté au-dessus de on.

L'addition l'économie du monde qu'il veut gouverner est faite avec deux signes de renvoi.

Fragment du folio 69

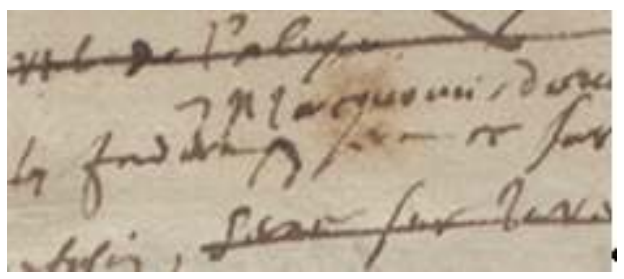

Cliché BNF

Texte final : Sur quoi les fondera on il l'économie du monde qu'il veut gouverner.

Dans les corrections, on lit dans l'interligne au-dessus de se régler à la justice qu'il ignore, la correction sur l'essentielle... Elle a été barrée ensuite. Même remarque chez Tourneur.

La scie : amorce de la science, sans doute.

Tout ce développement, qui forme une argumentation déjà serrée, est barré et remplacé par la formule placée en marge de droite: sera-ce sur la justice, il l'ignore. Abréviation très lapidaire d'un raisonnement sur l'essentielle justice.

Contrairement à l'apparence, ce n'est pas une réflexion sur la nature des lois. Le fragment touche le problème pratique de l'économie du monde : comment, étant reconnu que la loi naturelle ne nous est pas connue, organiser le monde ? Ce qui relève de la vanité, c'est la loi. Le problème pratique de l'économie du monde correspond à la misère, dans la mesure où il met au jour une impuissance.

Toutefois, l'idée de l'économie du monde qu'il veut gouverner est seconde : c'est un ajout. Pascal part des lois et vient après coup à l'idée de l'économie du monde. Problème : Qui est ce il?

\section{Justice}

Encyclopédie saint Augustin, Paris, Cerf, 2005, p. 834 sq. Justice.

BOUCHILLOUX Hélène, "Justice, force : les limites de la raison d'État selon Pascal », in ZARKA Y.C., Raison et déraison d'Etat, P.U.F., Paris, 1994, p. 341-357.

LAZZERI Christian, Force et justice dans la politique de Pascal, P.U.F., Paris, 1993. 
MESNARD Jean, « Pascal et la justice à Port-Royal », Commentaire, 121, printemps 2008, p. 163-173. La conférence « La justice à Port-Royal » prononcée par Jean Mesnard, lors de la Séance du lundi 16 janvier 2006 de l'Académie des Sciences Morales et Politiques est disponible sur Internet.

MOROT-SIR Édouard, Pascal, Presses Universitaires de France, Paris, 1973, p. 32 sq.

MOROT-SIR Édouard, «La justice de Dieu selon Pascal », in Justice et force. Politiques au temps de Pascal, Paris, Klincksieck, 1996, p. 281-295.

PÉCHARMAN Martine, «La justice selon Pascal », in G. Samama (dir.), La justice, Paris, Ellipses, 2001, p. 113-128.

NATOLI Charles M., "L'importance fondamentale de la justice dans l'apologétique de Pascal : le Dieu caché ", in Justice et force. Politiques au temps de Pascal, Paris, Klincksieck, 1996, p. 297-305.

SIMON Pierre-Henri, «Pascal, l'Histoire et la justice », in Le Jardin et la ville, Le Seuil, Paris, 1962, p. 46-58.

74 Voir la lettre d'Arnauld à Périer du 20 novembre 1669, in Pensées, éd. Luxembourg, Documents, p. 159-160. Le fragment visé est Laf. 520, qui ne figure pas dans l'édition de 1670.

«Ce 20 novembre [1669].

[...] J'espère que tout s'ajustera, et que, hors quelques endroits qu'il sera assurément bon de changer, on les fera convenir de laisser les autres comme ils sont; mais souffrez, monsieur, que je vous dise qu'il ne faut pas être si difficile, ni si religieux à laisser un ouvrage comme il est sorti des mains de l'auteur, quand on le veut exposer à la censure publique. On ne saurait être trop exact quand on a affaire à des ennemis d'aussi méchante humeur que les vôtres. Il est bien plus à propos de prévenir les chicaneries par quelque petit changement, qui ne fait qu'adoucir une expression, que de se réduire à la nécessité de faire des apologies. C'est la conduite que nous avons tenue touchant des considérations sur les dimanches et les fêtes, de feu M. de Saint-Cyran, que feu Savreux a imprimées. Quelques-uns de nos amis les avaient revues avant l'impression; et M. Nicole, qui est fort exact, les ayant encore examinées depuis l'impression, y avait fait faire beaucoup de cartons. Cependant les docteurs, à qui on les avait données pour les approuver, y ont encore fait beaucoup de remarques, dont plusieurs nous ont paru raisonnables et qui ont obligé à faire encore de nouveaux cartons. Les amis sont moins propres à faire ces sortes d'examen que les personnes indifférentes, parce que l'affection qu'ils ont pour un ouvrage les rend plus indulgents sans qu'ils le pensent, et moins clairvoyants. Ainsi, monsieur, il ne faut pas vous étonner, si ayant laissé passer de certaines choses sans en être choqués, nous trouvons maintenant qu'on les doit changer, en y faisant plus d'attention après que d'autres les ont remarquées. Par exemple, l'endroit de la page 293 me paraît maintenant souffrir de grandes difficultés, et ce que vous dites pour le justifier, que, selon saint Augustin, il n'y a point en nous de justice qui soit essentiellement juste, et qu'il en est de même de toutes les autres vertus, ne me satisfait point. Car vous reconnaitrez, si vous y prenez bien garde, que M. P. n'y parle pas de la justice, vertu qui fait dire qu'un homme est juste, mais de la justice quae jus est, qui fait dire qu'une chose est juste, comme : il est juste d'honorer son père et sa mère, de ne tuer point, de ne commettre point d'adultère, de ne point calomnier, etc. Or, en prenant le mot de justice en ce sens, il est faux et très dangereux de dire qu'il n'y ait rien parmi les hommes d'essentiellement juste; et ce qu'en dit M. Pascal peut être venu d'une impression qui lui est restée d'une maxime de Montagne, que les lois ne sont point justes en elles-mêmes, mais seulement parce qu'elles sont lois. Ce qui est vrai, au regard de la plupart des lois humaines qui règlent des choses indifférentes d'elles-mêmes, avant qu'on les eût réglées, comme 
que les aînés aient une telle part dans les biens de leurs père et mère ; mais très faux, si on le prend généralement, étant par exemple, très juste de soi-même, et non seulement parce que les lois l'ont ordonné, que les enfants n'outragent pas leurs pères. C'est ce que saint Augustin dit expressément de certains désordres infâmes, qu'ils seraient mauvais et défendus, quand toutes les nations seraient convenues de les regarder comme des choses permises. Ainsi, pour vous en parler franchement, je crois que cet endroit est insoutenable, et on vous supplie de voir parmi les papiers de M. Pascal, si on n'y trouvera point quelque chose qu'on puisse mettre à la place. Enfin, vous pouvez, monsieur, vous assurer que je travaillerai dans cette affaire avec tout le soin et toute l'affection qui me sera possible. Je salue Mlle Périer et tous vos enfants, et je m'estimerai toujours heureux de pouvoir faire quelque chose pour votre service $[. .$.$] »$

SFEZ Gérald, Les doctrines de la raison d'État, Paris, Colin, 200, p. 137 sq. Comme la justice absolue à laquelle les hommes aspirent, demeure inaccessible par la raison naturelle, et comme, après la chute, le moi se fait injustement centre de tout et veut être le tyran de tous les autres; la justice consiste à neutraliser cette tendance tyrannique de la libido dominandi, que l'on ne peut détruire en elle-même: p. 138. Selon Pascal la paix est la véritable définition de la justice, en raison de l'impossibilité de principe d'établir une justice, qui fait que l'ordre en tant qu'ordre est justice ; elle figure la justice de Dieu.

Certainement s'il la connaissait il n'aurait pas établi cette maxime, la plus générale de toutes celles qui sont parmi les hommes, que chacun suive les mours de son pays. L'éclat de la véritable équité aurait assujetti tous les peuples. Et les législateurs n'auraient pas pris pour modèle, au lieu de cette justice constante, les fantaisies et les caprices des perses et allemands.

77 La veri: tr. la vérité, correction de premier jet. Ou la véritable équité, comme dans la suite.

78 Convaincu a précédé assujetti, qui est une correction: Tourneur, dans son édition paléographique, lit convaincu. On a parfois lu communiqué. De convaincu à assujetti, s'effectue un passage de l'ordre des esprits à celui des corps. Assujetti semble aussi comporter aussi une idée de contrainte, voire de violence, qui est absente de convaincu.

Perses, Gascons, Allemands, Indiens : La référence 393 montre que Pascal a Montaigne sous les yeux. Gascon: il pense à Montaigne. Montaigne, II, XII, donnait Perses et Indes. La suppression des Gascons démontaignise le texte.

80 Perses : musulmans. Voir le trope d'Enésidème par les modes de vie et les coutumes, in Diogène LAËrCE, Vies des philosophes, IX, 83, éd. Goulet-Cazé, p. 1119 sq. «Les Perses ne jugent pas incongru de coucher avec leurs filles, les Grecs le jugent sacrilège... »

81 Allemands : protestants.

82 Laf. 81, Sel. 116. "Les seules règles universelles sont les lois du pays aux choses ordinaires et la pluralité aux autres. D’où vient cela? De la force qui y est. »

83 Laf. 86, Sel. 120. «Veri juris Nous n'en avons plus. Si nous en avions nous ne prendrions pas pour règle de justice de suivre les mœurs de son pays. C'est là que ne pouvant trouver le juste on a trouvé le fort, etc. »

84 Charron Pierre, De la sagesse, Livre I, ch. XVII, De l'imagination et opinion. "La vérité et l'être des choses n'entre ni ne loge chez nous de soi-même, de sa propre force et autorité : s'il était ainsi, toutes choses seraient reçues de tous, toutes pareilles et de même façon, sauf peu plus, peu moins ; tous seraient de même créance : et la vérité qui n'est jamais qu'une et uniforme, serait embrassée de tout le monde. Or il y a si grande 
diversité, voire contrariété d'opinions par le monde, et n'y a chose aucune de laquelle tous soient généralement d'accord, pas même le savants, et les mieux nés : qui montre que les choses entrent en nous par composition, se rendent à notre merci et dévotion, et logent chez nous comme il leur plaît, selon l'humeur et la trempe de notre âme ».

On la verrait plantée par tous les états du monde, et dans tous les temps, au lieu qu'on ne voit rien de juste ou d'injuste qui ne change de qualité en changeant de climat, trois degrés d'élévation du pôle renversent toute la jurisprudence, un méridien décide de la vérité.

MESNARD Jean, Les Pensées de Pascal, p. 188, et $2^{\mathrm{e}}$ éd., p. 197. Par rapport à la latitude de Paris, deux degrés d'élévation du pôle ne permettent pas de quitter la France; mais trois conduisent aux Pays-Bas ou en Angleterre.

Élévation du pôle : voir ReGis Pierre Sylvain, Cours entier de Philosophie, II, p. 9. «Ce qu'on appelle ainsi n'est que l'arc d'un méridien compris entre le Pôle et l'Horizon ». Voir aussi BRISson M., Dictionnaire raisonné de physique, t. I, 1781. On appelle arc d'élévation du pôle la portion du méridien qui renferme les degrés compris depuis le pôle jusqu'à l'horizon : plus cet arc est grand, plus le pôle est élevé.

SABRIÉ, De l'humanisme..., p. 289. Ni la philosophie ni les religions n'arrivent à s'entendre sur la vérité, les lois et les usages. «Ce qui est impie, injuste, abominable en un lieu est piété, justice, et honneur ailleurs; et ne saurais nommer une loi, coutume, créance reçue ou rejetée généralement partout ». Voir Sagesse, 1. 1, ch. XV.

\section{En peu d'années de possession les lois fondamentales changent, le droit à ses époques}

Port-Royal : ou peu d'années de possession. Les lois...

Laf. 61, Sel. 95. Justice. Comme la mode fait l'agrément aussi fait-elle la justice.

Année de possession est dans Montaigne, Essais, II, XII. «Et chez nous ici, j'ai vu telle chose qui nous était capitale, devenir légitime : et nous qui en tenons d'autres, sommes à mêmes, selon l'incertitude de la fortune guerrière, d'être un jour criminels de lèsemajesté humaine et divine, notre justice tombant à la merci de l'injustice: et en l'espace de peu d'années de possession, prenant une essence contraire ».

De possession: terme juridique? Sens? Puissance? Vigueur? Etablissement? Le Dictionnaire de Furetière ne donne ici aucun sens vraiment satisfaisant.

FonTETTE François, Vocabulaire juridique, Paris, P.U.F., 1988, p. 95. Possession : maîtrise de fait exercée sur un bien corporel avec l'intention d'exercer un droit réel. Possession d'état: situation de fait apparente qui laisse présumer la situation de droit correspondante et qui comporte trois éléments : 1 . nomen, c'est-à-dire le fait de porter le nom qui désigne cet état ; 2. tractatus, c'est le fait d'être traité comme ayant l'état dont il s'agit ; 3. fama, c'est le fait d'être considéré par le public comme ayant l'état en question.

L'entrée de Saturne au Lion nous marque l'origine d'un tel crime.

Avant crime, Pascal avait écrit droit.

Un tel crime: quel est le sens de tel? Simple identifiant? L'expression implique des connaissances partagées. Est-ce l'équivalent de tel crime?

Port-Royal supprime cette mention. Est-ce par souci de bienséance? 
$\mathrm{Au}$ temps de Pascal, Saturne est la plus lointaine planète connue, neuf fois et demie plus éloignée du Soleil que la Terre. Son cycle est de près de 30 ans : c'est la plus longue à parcourir son orbite, soit 29 ans et 167 jours. Sa révolution sur elle-même dure $10 \mathrm{~h} 15$.

La date d'entrée de Saturne au Lion est fixe. Mais l'éclipse du soleil prévue pour le 12 août 1654 devait se produire durant le passage du Soleil, de Saturne et de Mars dans le signe du Lion, ce qui était censé ne pas s'être produit depuis le Déluge. Un astrologue, le docteur Andreas, a publié des pronostics qui ont troublé les Parisiens: certains craignaient la fin du monde, tandis que d'autres s'en moquaient comme de superstitions. Claude Auvry, vicaire du cardinal Antoine Barberini, grand aumônier de France, demanda alors à Gassendi un écrit là-dessus. Celui-ci ne put se récuser. Les Sentiments sur l'éclipse qui doit arriver le 12. Du mois d'août prochain. Pour servir de réfutation aux faussetés qui ont été publiées sous le nom du Docteur Andreas, Paris, de l'imprimerie d'Antoine Vitré, MDCLIV, sont une brochure de 16 pages datée du 20 juillet. Le texte de cette réponse se trouve à la Bibliothèque de Clermont-Ferrand, R 1037, pièce XXXI, avec une note manuscrite, bien difficilement lisible, dont on suppose dit qu'elle est de Pascal. Voir $O C \mathrm{I}, \mathrm{p}$. 276, qui présente cette attribution comme une hypothèse. Selon $\mathrm{M}$. Le Guern, P. de Marca a aussi demandé un ouvrage à Pierre Petit, qui a écrit L'éclipse du soleil du 12 août 1654 ou raisonnements contre ses pronostiques, Paris, Alexandre Lesselin, 1654. Sur cette éclipse, voir LABROUSSE Élisabeth, L'entrée de Saturne au Lion. L'éclipse du Soleil du 12 août 1654, The Hague, Martinus Nijhoff, 1974 ; Pierre Gassendi. Sa vie, son œuvre, 1592-1655, p. 48 sq. Sur l'éclipse du soleil prévue pour le 12 août 1654, et la note de l'édition des Euvres complètes de Pascal par M. Le Guern, II, p. 1333.

Il faudrait rechercher l'événement de droit ou de crime qui a donné lieu à cette allusion. Il n'y en a peut-être pas: Pascal voudrait alors prendre cette conjonction astrologique comme exemple d'une manière fantaisiste de dater les événements humains.

\section{Plaisante justice qu'une rivière borne.}

Plaisante justice: voir CRoquetTe, Pascal et Montaigne, p. 119, n. 32. Montaigne donne crime.

Que le trajet d'une rivière rend injuste devient que le trajet d'une rivière rend crime, puis qu'une rivière borne.

Il s'agit sans doute d'une référence à Sel. 84, « Pourquoi me tuez-vous?»

Vérité au-deçà des Pyrénées, erreur au-delà.

Y. Maeda remarque que Pascal a d'abord voulu écrire monts; il a écrit, puis barré mo, et précisé par Pyrénées. Ces Pyrénées viennent de Montaigne.

Le mot montagne vient de Montaigne, qui écrit: "quelle vérité est-ce que ces montagnes bornent, mensonge au monde qui se tient au-delà ?» Pascal a barré le mot. Port-Royal, XXV, 5, donne une montagne. Port-Royal garde les Pyrénées. Suppression du mot crime.

Pour la France, on n'a pas le choix: ce sont les Alpes ou les Pyrénées. Au nord des Pyrénées, la France n'est pas pays d'Inquisition, contrairement à l'Espagne.

Formule à opposer à celle de la lettre à Fermat du 29 juillet 1654, OC II, p. $1137:$ « Je vois bien que la vérité est la même à Toulouse et à Paris ». Il est vrai que Toulouse est en deçà des Pyrénées. 
PINTARD René, Le libertinage érudit..., p. 507. Exemples de variations dans les mœurs tirés de La Mothe le Vayer. Voir les Dialogues d'Orasius Tubero, éd. 1716, t. 1, p. 30-42, 120-134, 135-160, 269-279. «Il n'y a rien de si frivole qui ne soit en quelque part très importante ; il n'y a folie, pourvu qu'elle soit bien suivie, qui ne passe pour sages ; il n'y a vertu qui ne soit prise pour un vice, ni vice qui ne tient lieu de vertu ailleurs. Voir aussi p. 536, sur La Mothe le Vayer: "étrange et ridicule morale, que les Alpes et les Pyrénées diversifient ». Soliloques sceptiques, Paris, Bilaine, 1670, p. 24. Il n'y a probablement pas à chercher de liaison entre Pascal et Le Vayer.

ARNAUld Antoine et Nicole Pierre, La logique, III, XX, a, 1, éd. Clair et Girbal, p. 261 sq.

Des sophismes d'amour-propre, d'intérêt et de passion, § 1 . «Nous jugeons des choses non par ce qu'elles sont en elles-mêmes; mais par ce qu'elles sont à notre égard; et la vérité et l'utilité ne sont pour nous qu'une même chose. Il n'en faut point d'autres preuves que ce que nous voyons tous les jours, que des choses tenues partout ailleurs pour douteuses, ou même pour fausses, sont tenues pour très certaines par tous ceux d'une nation ou d'une profession, ou d'un Institut: car n'étant pas possible que ce qui est vrai en Espagne, soit faux en France, ni que l'esprit de tous les Espagnols soit si différemment tourné de celui de tous les Français, qu'à ne juger des choses que par les règles de la raison, ce qui paraît vrai généralement aux une paraisse faux généralement aux autres; il est visible que cette diversité de jugement ne peut venir d'autre cause, sinon qu'il plaît aux unes de tenir pour vrai ce qui leur est avantageux, et que les autres n'y ayant point d'intérêt, en jugent d'une autre sorte. »

\section{Folio 365}

\section{Le B en tête du folio 365.}

Indication de Tourneur. Voir le A de Imagination.

Ils confessent que la justice n'est pas dans ces coutumes, mais qu'elle réside dans les lois naturelles communes en tout pays...

Barré : le for, selon Maeda. Fortune, peut-être, remplacé par hasard.

Point : barré ensuite de générale. C'est dans $\mathrm{C} 2$, mais barré.

Communes: d'autres, Maeda, Faugère, Brunschvicg, disent connues.

Voir plus haut la lettre d'Arnauld à Périer critiquant ce texte, du 20 novembre $1669,1^{\text {er }}$ R. M. ms. Guerrier, BN f. fr. 12988, p.80. Voir le texte in Pensées, éd. Lafuma, Luxembourg, Documents, p. 159-160. Pascal attaque à tort la justice quae jus est, et non pas seulement la justice qui fait qu'un homme est juste. Pascal s'est laissé influencer par Montaigne, mais on trouve une opinion correcte dans saint Augustin.

Travail texte en main de recueil des termes de Montaigne. Voir MESNARD Jean, La culture du XVII e siècle, p. 80. Comparaison avec la source de Montaigne.

Sur les lois naturelles, voir ci-dessous.

\section{Le larcin, l'inceste, le meurtre des enfants et des pères...}

MonTAIGNE, Essais, II, 12, Pléiade, p. 564. A partir de là, Pascal abandonne Montaigne, qui part dans des développements sur l'anthropophagie, p. 565 sq. Montaigne cite le meurtre des enfants, p. 564, des pères, la communication des femmes, le trafic de voleries, la licence à toutes sortes de voluptés. Mais il n'y a pas l'inceste chez Montaigne : il parle seulement des " mariages entre les proches... ", et cite Ovide sur la mère s'unit à son fils et le fils à la fille. Charron en revanche parle de l'inceste. Pascal 
barre tout ce qui a trait au sexe, y compris la communication des femmes, qui est dans Montaigne, mais il conserve l'inceste.

Voir le Trope d'Enésidème par les modes de vie et les coutumes, in DIOGENE LAËRCE, Vies, IX, 83, éd. Goulet-Cazé, p. 1119 sq. « Les Perses ne jugent pas incongru de coucher avec leurs filles, les Grecs le jugent sacrilège... »

Busson Henri, La religion des classiques, p. 217. Cite Gilbert, Arie et Petus, ou les amours de Néron, 1660, achevé d'imprimer le 2 décembre 1659. C'est Pétrone qui parle à Sénèque :

«Ce qu'on croit juste à Rome ailleurs est injustice.

La vertu d'un pays dans un autre est un vice.

Chaque peuple a ses mœurs, ses coutumes, ses lois.

(...) Parmi les rois de Perse on approuve l'inceste,

A Sparte le larcin est un vice permis ».

GASSENDI Pierre, d'après PINTARD R., Le libertinage érudit..., p. 480. Coutumes opposées des peuples sur le mariage ; célibat ici, polygamie là ; union indissoluble ici, prostitution là ; voir Opera, III, p. 102, p. 195-197. Seule paraît universelle la recherche épicurienne d'un souverain bien consistant dans la volupté.

PINTARD René, Le libertinage érudit.., p. 480. Voir p. 507 sur La Vayer, Dialogues d'Orasius Tubero, éd. 1716, t. 1, p. 42-49 : le larcin était honorable en Cilicie, en Égypte, en Grèce ; mensonge, prostitution ont été honorés ou le sont encore.

Le meurtre des enfants : avortement, exposition.

Le meurtre des pères : le parricide est le crime irrémissible dans la tragédie. Voir ce qu'en dit Domat Jean, Traité des lois, I, p. II. Les Romains s'étaient donné, « comme les autres peuples, la licence d'ôter la vie et à leurs esclaves, et à leurs propres enfants. Comme si la puissance que donnent la qualité de père et celle de maître, pouvait dispenser des lois de l'humanité ». Domat poursuit : "cette opposition si extrême entre l'équité qui luit dans les lois si justes qu'ont fait les Romains, et l'inhumanité de cette licence, fait bien voir qu'ils ignoraient les sources de la justice même qu'ils connaissaient, puisqu'ils blessaient si grossièrement par ces lois barbares, l'esprit de ce principe, qui sont les fondements et tout ce qu'il y a de justice et d'équité dans leurs autres lois. »

FERREYROLles Gérard, Pascal et la raison du politique, p. 183. Recours à l'hyperbole.

131 FERREYROLLES Gérard, «Les païens dans la stratégie argumentative de Pascal », in Pascal. Religion, Philosophie, Psychanalyse, Revue philosophique de la France et de l'étranger, $\mathrm{n}^{\circ} 1$, janv.-mars 2002, p. 31.

Dans les Provinciales, Pascal soutient que le meurtre est contre la nature. Voir Provinciale XIV, 1-2. «Qu'y a-t-il de plus naturel que ce sentiment qu'un particulier n'a pas droit sur la vie d'un autre? Nous en sommes tellement instruits de nous-mêmes, dit saint Chrysostome, que, quand Dieu a établi le précepte de ne point tuer, il n'a pas ajouté que c'est à cause que l'homicide est un mal ; parce, dit ce Père, que la loi suppose qu'on a déjà appris cette vérité de la nature. Aussi ce commandement a été imposé aux hommes dans tous les temps. L'Évangile a confirmé celui de la loi, et le Décalogue n'a fait que renouveler celui que les hommes avaient reçu de Dieu avant la loi, en la personne de Noé, dont tous les hommes devaient naître; car dans ce renouvellement du monde, Dieu dit à ce patriarche : Je demanderai compte aux hommes [de la vie des hommes,] et au frère de la vie de son frère. Quiconque versera le sang humain, son sang sera répandu ; parce que l'homme est créé à l'image de Dieu. »

Se peut-il rien de plus plaisant qu'un homme ait droit de me tuer... 

bornée par une rivière. pas. démence exécrable ».

Sans doute : assurément, certainement. Voir CRoQuetTe, Pascal et Montaigne, p. 16. p. $10, \mathrm{n}^{\circ}$ XIV. nécessaires

Voir Laf. 20, Sel. 54, et Laf. 51, Sel. 84, sur le même thème. Voir plus haut, sur la justice

MesnaRd Jean, "Achèvement et inachèvement dans les Pensées de Pascal ", Studi francesi, 143, anno XLVIII, maggio-agosto 2004, p. 300-320. Voir p. 308. Ironie de la représentation de la frontière par l'eau, qui, s'écoulant sans cesse, est comme n'existant

VolTAIRE, Dernières remarques, XLVII : "Plaisant n'est pas le mot propre; il fallait

\section{Il y a sans doute des lois naturelles, mais cette belle raison corrompue a tout corrompu.}

Pascal écrit d'abord cette belle raison dogmat, puis il corrige en corrompue.

Corrections. Examiné renvoie à dogmatique. Gâté prolonge corrompu.

MonTAIGNe, Essais, II, 12, éd. Rat, Garnier, I, p. 653 ; éd. Pléiade, p. 564. « Il est croyable qu'il y a des lois naturelles, comme il se voit ès autres créatures; mais en nous elles sont perdues, cette belle raison humaine s'ingérant partout de maîtriser et commander, brouillant et confondant le visage des choses selon sa vanité et inconstance. Nihil itaque amplius nostrum est : quod nostrum dici, artis est » (citation de CICÉRON, De finibus, V, 21).

Domat Jean, Lois civiles, p. LXXX : il n'y a en fait de lois naturelles et immuables que celles qui viennent de Dieu; les lois humaines sont des lois positives et arbitraires, parce que les hommes peuvent les instituer, les établir, les changer et les abolir. Les lois immuables sont naturelles, tellement justes toujours et partout qu'aucune autorité ne peut les changer ni les abolir. Ce sont celles qui ne peuvent être changées sans porter atteinte aux deux lois fondamentales de l'amour des hommes, p. LV sq. Voir p. LVII, des exemples de lois immuables. Les lois immuables tirent leur origine du fait que ce ne sont que des extensions des deux lois fondamentales du Décalogue ; c'est de là qu'elles tirent leur justice essentielle. Les lois naturelles : p. LXXIV. Ces lois règlent le passé et l'avenir sans qu'on les publie. Sur la manière dont elles règlent le passé : p. $9, n^{\circ}$ XII ; et

Mais la loi est générale, et ne peut déterminer toutes les circonstances; on remédie à ce manque par des lois arbitraires, qui peuvent être très différentes d'un lieu à un autre.

Les lois arbitraires sont d'origine humaine. Voir Domat Jean, Lois civiles, p. LVI sq. : Ce sont celles qu'une autorité légitime peut établir, changer, abolir selon le besoin, sans violer l'esprit des lois fondamentales ni blesser les principes de la société. Elles sont

1. pour régler certaines difficultés nées de l'application des lois immuables, notamment sur l'héritage, la propriété, la majorité, juste prix, p. LVIII-LX ;

2. parce que certains usages existent dans la société qui ont été inventés.

Ces lois sont de deux sortes : certaines lois arbitraires sont des suites des lois naturelles, d'autres ne servent qu'à régler des matières indifférentes : $p$. LXIII. Les lois arbitraires sont indifférentes aux fondements de la société ; leur justice consiste dans l'utilité particulière, selon temps et lieux. Les lois arbitraires ne règlent que l'avenir, après leur publication. Etant d'origine humaine, elles peuvent être abolies par les hommes. 
ADoRno Francesco, « Pascal et le droit naturel », in Les Pascal à Rouen, 1640-1648, Rouen, Publications de l'Université de Rouen, 2001, p. 357-374. L'homme ne connaît pas la justice naturelle, mais cela ne signifie pas que les lois naturelles n'existent pas. Nous n'avons plus de lois naturelles n'est pas synonyme de il n'y a pas de lois naturelles: p. 358. Problème : peut-on, à partir des restes de lumière laissés par le péché originel dans l'esprit de l'homme, postuler la possibilité d'une présence dans l'âme des principes du droit naturel dont la connaissance peut en quelque sorte être améliorée?

Sellier Philippe, Pascal et saint Augustin, Paris, Colin, 1970, p. 90 sq. Loi naturelle et obscurcissement chez saint Augustin. Loi naturelle et obscurcissement chez Pascal: p. 93 sq.

MontaIGNe, Essais, II, 30, éd. Rat, Garnier, I, p. 225, éd. Pléiade, p. 195. «Comme si nous avions l'attouchement infect, nous corrompons par notre maniement les choses qui d'elles-mêmes sont belles et bonnes. Nous pouvons saisir la vertu de façon qu'elle en deviendra vicieuse, si nous l'embrassons d'un désir trop âpre et violent ».

La première citation vient des Essais, II, 12 ; après quoi Pascal saute brusquement au $3^{\mathrm{e}}$ tome. Sur le manuscrit, les citations sont écrites au fil du texte, sans renvoi, et dans le corps de la page centrale. Il n'est pas pensable qu'elles aient été destinées à rester telles quelles ; peut-être devaient-elles fournir des développements nouveaux.

Nihil amplius nostrum est, quod nostrum dicimus artis est.

151 Nihil itaque amplius nostrum est : quod nostrum dici, artis est : Pas d'itaque, et dico au lieu de dici. Difficile de traduire. CICÉRON, De finibus, V, $21:$ « Car il ne reste rien qui soit nôtre : ce que nous appelons nôtre est l'effet de l'art ». Ce n'est pas le sens de Cicéron, où ce sont deux phrases, dont la fin de la première est accrochée au début de la seconde. Cicéron parle de tout autre chose: ce qu'il y a chez l'homme de plus noble et de meilleur, la nature l'a laissé en plan. Mais de la vertu même, elle a tracé une ébauche, pas davantage, nihil amplius. Itaque nostrum est, c'est donc à nous de faire ce travail que la nature n'a pas fait; "quod nostrum dico artis est », quand je dis-nous, c'est la philosophie, dit Cicéron en incise. Cicéron réfléchit sur ce qui revient à la nature et ce qui nous revient à nous. Montaigne sert de filtre entre Cicéron et Pascal. Cicéron dit le contraire de Pascal : il y a bien du nôtre selon Cicéron.

Voir Montaigne, Essais, II, 12, éd. Pléiade, p. 564. «Il est croyable qu'il y a des lois naturelles, comme il se voit ès autres créatures; mais en nous elles sont perdues, cette belle raison humaine s'ingérant partout de maîtriser et commander, brouillant et confondant le visage des choses selon sa vanité et inconstance. Nihil itaque amplius nostrum est : quod nostrum dici, artis est. » Dans l'édition de 1652, la citation de Montaigne n'est pas référencée. Traduction française de l'édition de 1652 : « Il ne reste plus rien qui soit vraiment à nous. Ce qu'on dit à nous est à l'art». Pourtant, la référence est habituellement dans la marge. Qui traduit dans cette édition? Curieux : Pascal note le latin, et non la traduction qui se trouve dans la marge. Voir CRoQuetTe, Pascal et Montaigne, p. 16.

\section{Ex senatus-consultis et plebiscitis crimina exercentur}

Pas de retouche.

155 SÉnÈQUE, Epîtres, 95: "C'est en vertu des senatus-consultes et des plébiscites qu'on commet des crimes ». Cité par Montaigne, Essais, III, 1. Traduction dans Montaigne, 1652 : «Les méchancetés s'exercent par les arrêts du sénat et par les ordonnances du peuple ». Le texte original, dans Montaigne est « Ex senatusconsultis plebisquescitis scelera 
exercentur ", Essais, III, 1, éd. Thibaudet et Rat, Pléiade, p. 774, éd. Balsamo, Pléiade, p. 836. Noter que scelera est le texte donné par les éditions des Lettres à Lucilius du XVI ${ }^{e}$ siècle, alors que les éditions modernes des Lettres à Lucilius, XCV, 30, donnent saeva. scelera exercentur.» utilisés).

RO, 397-1

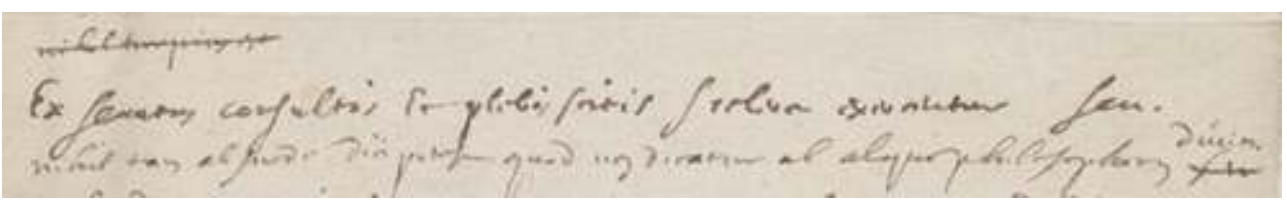

Charron donne la phrase de Sénèque dans Sagesse, I.

Pascal a visiblement été frappé par cette citation, car elle revient à plusieurs reprises dans les Pensées. Peut-être que c'est en composant un dossier en vue des Provinciales que la phrase de Sénèque a retenu son attention.

L'apparition la plus ancienne doit être Laf. 962, Sel. 796. «Ex senatus consultis et plebiscitis. Demander des passages pareils. » La citation est incomplète. L'ensemble du texte de Laf. 962, Sel. 796 comprenant cet extrait appartient aux notes pour les Provinciales, précisément pour la préparation de la $\mathrm{XV}^{\mathrm{e}}$ Provinciale, sur papier du type cadran et B cœur C Voir l'Album de Pol Ernst, p. 150 (ce papier est l'un des premiers

Mais sur le manuscrit, cette citation se trouve entourée d'une ligne circulaire, à droite d'un texte qui porte sur des accusations d'hérésie lancées contre Pascal, au nom des décisions prises par Rome contre les cinq propositions de Jansénius : " Je ne suis point hérétique. Je n'ai point soutenu les 5 propositions. Vous le dites et ne le prouvez pas. Je dis que vous avez dit cela et je le prouve. » A gauche on lit aussi « Vous me menacez ».

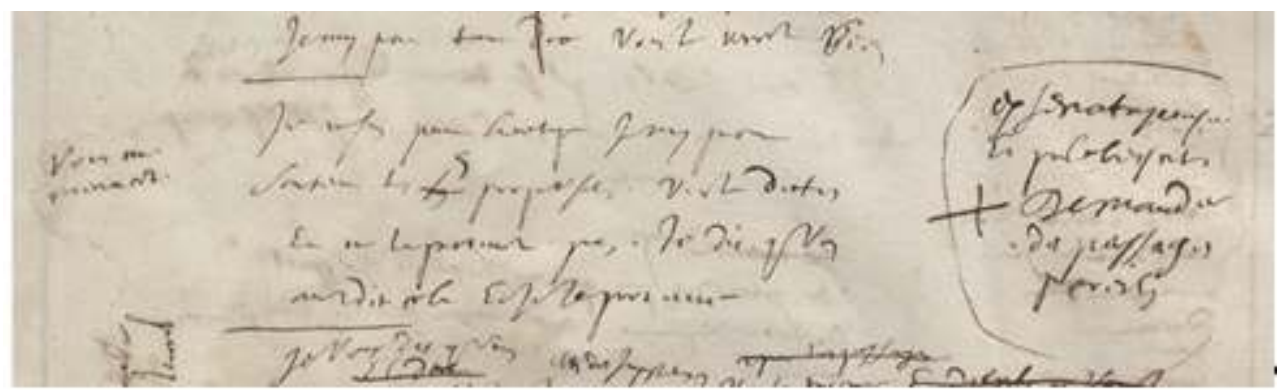

Ce serait vers cette même époque que Pascal aurait cherché des passages pareils. Ces passages pareils seraient dans Laf. 506-507-508, Sel. 673-679. Pascal a été les chercher directement dans les Essais.

On trouve la citation de Sénèque dans Laf. 507, Sel. 675. « Ex senatusconsultis et plebiscitis

RO, 214-3

Noter que le manuscrit ne donne pas le numéro 588 qu'on lit dans l'édition Lafuma Luxembourg, p. 341. Le volume de Notes, p. 96, indique que la référence 588, qui renvoie à l'édition des Essais de 1652, est portée sur la Copie. Selon Lafuma, cette référence a disparu "lorsque le papier a été rogné ». Cette affirmation est soutenable, à condition de ne pas chercher le 588 à droite, car le papier découpé comportant une large place, qui aurait été suffisante pour marquer 588. Mais si l'on regarde la Copie C2, p. 406, on 
trouve le 588 dans la marge à gauche. Il est tout à fait possible que cette référence ait été portée sur la gauche du papier du manuscrit, et qu'elle ait été supprimée lors du découpage.

Copie C2, p. 406

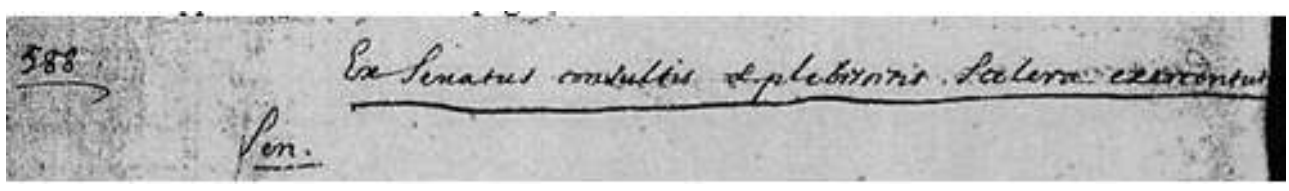

Et en effet, un peu plus haut, dans le fragment Laf. 506, Sel. 674, on voit « 583. Nae iste magno conatu magnas nugas dixerit. Terent. " : La référence 583 est visible sur le manuscrit (RO, 269-3), quoique le 5 ait presque complètement disparu au découpage. Cela suppose que ce découpage n'a pas été effectué par Pascal, car comme la référence figure sur la Copie, cette dernière a nécessairement précédé le découpage. Or la Copie a été faite après la mort de Pascal.

Fragment Laf. 506, Sel. 674

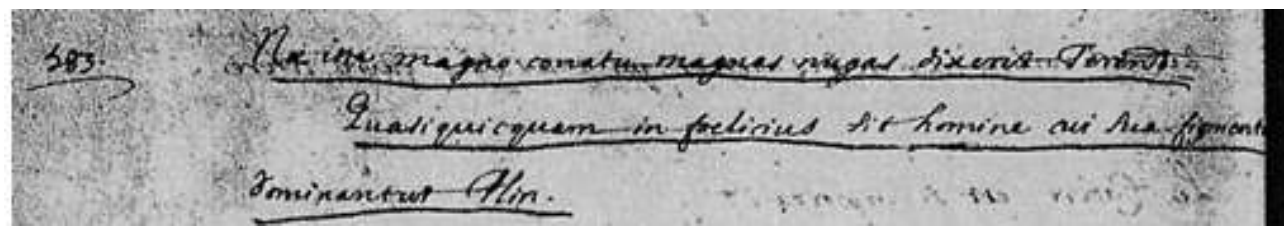

Pascal note le texte, avec la référence des numéros de pages de l'édition des Essais de 1652 que Pascal utilise. Ce fragment donne bien Scelera au lieu de crimina. Pascal transforme pourtant l'original, puisque Montaigne donne plebisquescitis, et que Pascal note et plebiscitis. Il ne semble pas que ce papier figure dans les relevés de Pol Ernst.

La citation est enfin reprise dans Laf. 60, Sel. 94, sous la forme : Ex senatus-consultis et plebiscitis crimina exercentur, qui substitue crimina à scelera.

Crimina, en principe, signifie chef d'accusation. Mais il faut bien remarquer que ce sens est le plus proche des préoccupations de Pascal au moment où il a commencé à chercher des «passages pareils » : la XV $\mathrm{X}^{\mathrm{e}}$ Provinciale porte sur la calomnie, c'est-à-dire sur des chefs d'accusation, plutôt que sur des crimes à proprement parler. On serait donc là en présence d'une persistance des préoccupations du temps des Provinciales au sein même du texte des Pensées. D'autre part, crimina doit bien être entendu dans Laf. 60, Sel. 94, au sens de grief, de chef d'accusation, et non au sens de crime.

\section{Ut olim vitiis sic nunc legibus laboramus.}

TACITE, Annales, III, 25 : « Nous souffrions jadis de nos vices, maintenant de nos lois »; cité par Montaigne, Essais, III, 13. Montaigne cite ut olim flagitiis, sic nunc legibus laboramus. Tacite donne, d'après l'édition de Juste Lipse utilisée par Montaigne : utque antehac flagitiis, ita tunc legibus laborabatur. Le laborabatur s'explique par le caractère historique du texte.

Pas de traduction française en Montaigne 1652, mais la référence est fausse, Tacite, Annales IV. Montaigne fait du récit au passé une constatation sur l'état des choses auquel participe le locuteur : ut olim flagitiis, sic nunc legibus laboramus. Chez Montaigne, cela ne désigne qu'un extrême, alors que chez Pascal, c'est l'essentiel. 
Pascal ne change qu'un mot de Montaigne, vitiis remplace flagitiis.

Pas de retouche.

\section{Sens de ces citations dans Montaigne et dans Pascal}

Intérêt de la mention chercher des passages pareils : travail de recherche et de sélection des citations.

Montaigne effectue une confrontation de la justice positive et de la naturelle. Chez Pascal, le défaut des lois positives prouve l'absence des lois naturelles.

De cette confusion arrive que l'un dit que l'essence de la justice est l'autorité du législateur, l'autre la commodité du souverain, l'autre la coutume présente, et c'est le plus sûr.

L'un barré, puis revient un peu plus bas.

L'autorité du législateur: MONTAIGNE, Essais, II, XII : "Protagoras et Ariston ne donnaient autre essence à la justice des lois, que l'autorité et opinion du législateur : et que cela mis à part, le bon et l'honnête perdaient leurs qualités, et demeuraient des noms vains, de choses indifférentes. Thrasymachus en Platon estime qu'il n'y a point d'autre droit que la commodité du supérieur. »

La commodité du souverain : doctrine machiavélienne.

Voir Romilly Jacqueline de, Les grands sophistes..., p. 158. La portée destructrice de la distinction entre physis et nomos. Thrasymaque et l'idée que la justice est l'intérêt des plus forts : p. 162. Qui fait les lois, sinon les gouvernants? Les législateurs pensent à leur propre intérêt.

La coutume présente. Les coutumes sont le plus bas degré des lois ; voir ci-dessous. Voir MONTAIGNE, Essais, II, 12, éd. Pléiade, p. 562. Montaigne fournit l'argument initial : la seule loi générale c'est d'obéir aux lois de son pays. "C'est la règle des règles, et générale loi des lois, que chacun observe celles du lieu où il est ", suit une sentence grecque de Crispin. L'idée vient de Socrate, selon XÉNOPHON, Mémorables, I, III.

La justice est l'obéissance à la loi établie dans la cité dans laquelle on vit : voir DUMONT Jean-Paul, Les sophistes, p. 174. Fragment d'Antiphon le sophiste sur la politique : « La vertu de justice consiste à ne pas transgresser ce que la cité dans laquelle on vit comme citoyen, considère comme légal.» Mais Antiphon ajoute qu'il en découle que si l'on transgresse les lois à l'insu des concitoyens, il échappe à toute infamie et à tout châtiment : p. 175.

CHARRon Pierre, Sagesse, II, 8. "Or l'advis que je donne ici à celui qui veut être sage est de garder et observe de parole et de fait les lois et coutumes que l'on trouve établies au pays où l'on est. »

Voir la première maxime de la morale provisoire de Descartes dans le Discours de la méthode, III, § 2. «La première était d'obéir aux lois et aux coutumes de mon pays, retenant constamment la religion en laquelle Dieu m'a fait la grâce d'être instruit dès mon enfance, et me gouvernant en toute autre chose suivant les opinions les plus modérées et les plus éloignées de l'excès qui fussent communément reçues en pratique par les mieux sensés de ceux avec lesquels j'aurais à vivre. »

LAComBe Roger, L'apologétique de Pascal, p. 148 sq. Deux types de réponses possibles : 1. les divergences ne sont pas bien nettes, et correspondent non à des contrariétés essentielles, mais à des adaptations ; c'est la théorie des climats ; on peut alors soutenir 
que les lois en vigueur traduisent diversement une même justice selon les circonstances ; 2. celle qui consiste à dire que les valeurs des civilisations supérieures de l'occident qui se sont répandues représentent des aspirations supérieures à peu près universelles.

Seule réponse qui porte : il est faux que si la véritable justice était connue, elle se serait imposée d'elle-même universellement. C'est à quoi tend la doctrine de l'idéologie : on suppose que même quand la vérité est connue, l'armature idéologique est telle que le peuple ne la voit pas.

\section{Rien suivant la seule raison n'est juste de soi, tout branle avec le temps.}

Rien suivant la seule raison n'est juste de soi : la première version est Rien n'est plus juste de soi ; puis : Rien n'est plus juste de soi-Le tem ; puis : Rien n'est plus juste de soi le tem. Enfin, la rédaction définitive.

Tout branle avec le temps : premier jet, tout branle avec le mo : sans doute le moment. Peutêtre le mouvement?

La coutume est toute l'équité, par cette seule raison qu'elle est reçue. C'est le fondement mystique de son autorité. Qui la ramènera à son principe l'anéantit.

Les a précédé la selon Maeda. Mais ce n'est pas évident.

Le font est resté ; Pascal n'a pas corrigé en fait, comme l'aurait voulu le sujet La coutume.

La coutume: du sens juridique, on passe au sens philosophique.

Equité n'est pas équivalent de justice.

Fondement mystique est de Montaigne.

\section{Coutume, coutumes}

Domat Jean, Les lois civiles, p. LXXXVI. Les coutumes ne sont pas écrites, mais se sont établies, soit par le consentement des peuples, et par une sorte de convention de les observer, soit par un usage insensible qui les a autorisées. Les lois écrites et les coutumes : p. 8. Les coutumes tirent leur autorité du consentement universel : p. 9.

LEMAITRE Antoine, Plaidoyers, XII, p. 235 sq. Nature des coutumes. Egalité des coutumes entre elles, mais prééminence de la coutume de Paris. Le fondement des coutumes est la seule volonté des peuples, ce qui explique leur diversité.

WENDROCK, Provinciales, I, éd. De 1700, p. 315. Les lois l'emportent sur les coutumes.

CHEVALIER Jacques, Pascal, Paris, Plon, 1922, p. 221 sq. La coutume.

McKENNA Antony, «Coutume/Nature : la fortune d'une pensée de Pascal », Équinoxe, n 6, été 1990, p. 83-98.

FERREYROLLES Gérard, "Pascal critique de la coutume ", Équinoxe, n 6, été 1990, p. 99-115.

FERREYROLLES Gérard, Les reines du monde. L'imagination et la coutume chez Pascal, Paris, Champion, 1995.

PARMENTIER Bérengère, Le siècle des moralistes, Paris, Seuil, 2000, p. 272 sq. Politique de la coutume.

Trope sceptique d'Enésidème par les modes de vie et les coutumes 
LONG et SEDLEY, Les philosophes hellénistiques, III, Les Académiciens. La renaissance du pyrrhonisme, p. 71-72.

SEXTUS EMPIRICUS, Esquisses pyrrhoniennes, I, 145 sq., éd. P. Pellegrin, p. 133 sq.

DIOGENE LAËRCE, Vies, IX, 83, éd. Goulet-Cazé, p. 1119 sq.

MERSENNE, La vérité des sciences, I, p. 34 sq. « La Morale n'est pas moins douteuse, car on fait une infinité de chimères sur ce principe, bonum amplectendum, malum fugiendum est, l'un disant qu'une chose est bonne, et l'autre qu'elle est mauvaise: un troisième viendra, qui la tiendra pour indifférente, car un chacun suit le plus souvent son affection, quand il juge des choses morales. »

Rien n'est si fautif que ces lois qui redressent les fautes. Qui leur obéit parce qu'elles sont justes, obéit à la justice qu'il imagine, mais non pas à l'essence de la loi. Elle est toute ramassée en soi. Elle est loi et rien davantage.

Elle est toute en soi : premier jet.

MONTAIGNE, Essais, III, 13, éd. Pléiade, p. 1049-1050. « Or les lois se maintiennent en crédit, non par ce qu'elles sont justes, mais par ce qu'elles sont lois. C'est le fondement mystique de leur autorité : elles n'en ont point d'autre. Qui bien leur sert. Elles sont souvent faites par des sots. Plus souvent par des gens, qui en haine d'équalité ont faute d'équité : Mais toujours par des hommes, auteurs vains et irrésolus. » Le texte suit Montaigne de près; mais Pascal, là aussi, accentue l'ironie de la plaisanterie: Montaigne écrit qu'il «n'est rien si lourdement et largement fautier que les lois, ni si ordinairement "; Pascal précise " ces lois qui redressent les fautes ». Manière de dire que c'est en ce qu'elles prétendent redresser les fautes que les lois sont fautives, en ce qu'elles veulent être bonnes qu'elles sont mauvaises. Pascal passe à une idée différente : il parle non de l'autorité des lois, mais de leur essence. Processus de généralisation.

CROQUETTE Bernard, Pascal et Montaigne, p. 19, n 8. Passage de Charron qui a pu servir de relais vers Montaigne.

Laf. 66, Sel. 100. «Injustice. Il est dangereux de dire au peuple que les lois ne sont pas justes, car il n'y obéit qu'à cause qu'il les croit justes. C'est pourquoi il faut lui dire en même temps qu'il y faut obéir parce qu'elles sont lois, comme il faut obéir aux supérieurs non pas parce qu'ils sont justes, mais parce qu'ils sont supérieurs. Par-là voilà toute sédition prévenue, si on peut faire entendre cela et que proprement c'est la définition de la justice. »

3 Laf. 645, Sel. 530. « La justice est ce qui est établi. Et ainsi toutes nos lois établies seront nécessairement tenues pour justes sans être examinées, puisqu'elles sont établies. »

FERREYROLLES Gérard, Pascal et la raison du politique, p. 186. Exactitude de la définition pour la loi positive, qui selon les juristes, est juste parce qu'elle est loi : p. 188.

CHINARD Gilbert, En lisant Pascal, Lille, Giard, Genève, Droz, 1948, p. 70 sq. Rapprochement avec Hobbes, De cive, XII.

Qui voudra en examiner le motif le trouvera si faible et si léger...

Voir Laf. 44-45, Sel. 78, l'imagination donne du poids à des choses insignifiantes.

L'art de fronder, bouleverser les états...

L'art de bouleverser les états et de fronder : premier jet.

L'art de fronder : fronder est barré dans $\mathrm{C}_{1}$, et Port-Royal, $\mathrm{XXV}, 6$, supprime fronder, pour donner de reprendre les lois fondamentales. 

1986, p. 67 sq. Les lois fondamentales relatives à la couronne et au domaine sont une norme juridique supérieure établie au-dessus du roi. Elles présentent trois caractères. Elles sont constitutionnelles: leur finalité est de gérer de façon intangible l'organisation des pouvoirs au sommet de l'État; elles ne sont pas formulées dans un document solennel, comme dans nos constitutions; mais il s'agit bien d'une constitution coutumière. Elles sont traditionnelles, car l'idée d'une norme supérieure s'imposant au roi est très ancienne; elle remonte à la promesse du sacre. Elles sont 
limitatives de l'autorité royale; le Parlement de Paris en est le gardien. À de nombreuses reprises, il sanctionne des actes royaux qui y contreviennent. Les autres cours souveraines se disent unies au Parlement de Paris pour assurer la garantie des lois fondamentales. La transmission de la couronne de France était déjà organisée autour d'un ensemble de règles apparues progressivement $d u \mathrm{X}^{\mathrm{e}}$ au XIV $\mathrm{X}^{\mathrm{e}}$ siècle. À la fin du XVIe siècle, un dernier principe est posé à l'occasion de l'accession de Henri IV au trône : le roi de France doit être catholique.

CABOURDIN Guy et VIARD Georges, Lexique historique de la France d'Ancien Régime, Paris, Armand Colin, 1978, p.195. Les lois fondamentales sont un ensemble de règles coutumières qui limitent la souveraineté absolue du roi et qu'il ne peut modifier. Elles tournent autour des deux notions de la continuité de l'Etat et de l'indépendance de la couronne. Ces règles sont: 1 . que le gouvernement de la France est une monarchie héréditaire, par ordre de primogéniture ; 2 que les femmes, leurs descendants et les bâtards sont exclus de la couronne (loi salique) ; 3. la majorité des rois est fixée à 13 ans révolus (ordonnance de 1374); pendant la minorité, la régence est assurée par la reine mère ou à défaut par le premier prince du sang; 4 . le successeur légitime est considéré comme roi dès la mort du prédécesseur ; 5 . par les serments du sacre, le roi s'engage vis-à-vis des évêques de France à leur conserver leurs privilèges canoniques, leurs lois et leur justice et vis-à-vis du peuple de France à le protéger, le garder en paix et justice ; 6. le principe de catholicité; 7. le domaine royal est inaliénable; 8. la puissance temporelle est indépendante à l'égard du pouvoir spirituel.

ReTZ, Mémoires, II, Euvres, éd. Hipp, Pléiade, p. 193 sq. « Il y a plus de douze cents ans que la France a des rois ; mais ces rois n'ont pas toujours été absolus au point qu'ils le sont. Leur autorité n'a jamais été réglée, comme celle des rois d'Angleterre et d'Argon, par des lois écrites. Elle a été seulement tempérée par des coutumes reçues et comme mises en dépôt, au commencement dans les mains des États généraux, et depuis dans celle des parlements. Les enregistrements des traités faits entre les couronnes et les vérifications des édits pour les levées d'argents sont des images presque effacées de ce sage milieu que nos pères avaient trouvé entre la licence des rois et le libertinage des peuples. Ce milieu a été considéré par les bons et sages princes comme un assaisonnement de leur pouvoir, très utile même pour le faire goûter aux sujets ; il a été regardé par les malhabiles et par les mal intentionnés comme un obstacle à leurs dérèglements et à leurs caprices. " Voir p.1299-1300, n. 3, sur la définition des lois fondamentales.

CARRIER Hubert, Le labyrinthe de l'État. Essai sur le débat politique en France au temps de la Fronde (1648-1653), p. 161 sq. Nature et étendue de la puissance royale. La Fronde et le problème constitutionnel: p. 161 sq. La constitution coutumière de la monarchie traditionnelle ne détermine qu'avec imprécision la frontière entre l'affirmation de la volonté royale et le droit de résistance des cours souveraines; c'est selon les contemporains une bonne chose qu'il y ait un mystère de l'État, car il y aurait danger à fixer une fois pour toutes, à institutionnaliser l'équilibre résultant d'un perpétuel compromis entre la puissance royale et les droits du peuple. Voir p. 165, un passage de la réponse de Molé à la reine sur la nécessité de préserver ce mystère. Retz en parle dans ces termes : voir p. 166, sur le fait que, comme il raisonne en homme d'action et non en théoricien, il ne cherche pas à donner une vision abstraite, cohérente et rationnelle des forces qui composent l'État; il s'en fait une image pratique, le comparant à une machine aux rouages compliqués, voire à un organisme vivant. 
MONGRÉDIEN Georges, La vie littéraire au XVII siècle, p. 56 sq. Pamphlétaires qui, à partir de 1651, rappellent au roi les « lois fondamentales » du royaume et le « contrat » tacite passé entre le monarque et son peuple, incompatible avec le despotisme d'un Richelieu et la tyrannie d'un Mazarin.

ARNAULD D'ANDILLY Robert, Avis d'État, 1649, p. 23. «L'intérêt du roi consiste à ne point subvertir les anciens ordres, et à maintenir les lois fondamentales de son empire, étant indubitable que la monarchie ayant été fondée et subsistant sur ces vieilles maximes, il faut ne s'en point départir pour la faire durer. Et c'est une erreur de croire qu'un prince légitime et bien reconnu par ses sujets relève son autorité en détruisant les établissements anciens qui ont fait subsister ses prédécesseurs » (cité d'après CARRIER Hubert, « Port-Royal et la Fronde »).

CARRIER Hubert, Le labyrinthe de l'État. Essai sur le débat politique en France au temps de la Fronde (1648-1653), p. 194 sq. Voir ci-dessous la note sur II, 39. De nombreuses mazarinades soulèvent le problème de la soumission $\mathrm{du}$ roi aux lois fondamentales $\mathrm{du}$ royaume.

CHINARD Gilbert, En lisant Pascal, Lille, Giard, Genève, Droz, 1948, p.70 sq. Rapprochement avec Hobbes, De cive, XII.

C'est un jeu sûr pour tout perdre; rien ne sera juste à cette balance.

C'est un jeu sûr pour tout perdre: expression qui appartient au langage du jeu et du pari. Le fragment Laf. 418, Sel. 680, donne au contraire un jeu sûr pour tout gagner.

Balance: dans un texte sur la justice.

\section{Quatrième page, verso de la troisième page, folio $n^{\circ} 366$}

\section{Remarques sur la partie non barrée du texte}

\section{Cependant le peuple prête aisément l'oreille à ces discours...}

MonTAIGNe, Essais, I, XXIII, éd. Garnier, I, p. 125, éd. Pléiade, p. 117 sq. «Il y a grand doute, s'il se peut trouver si évident profit en changement d'une loi reçue, telle qu'elle soit, qu'il y a de mal à la remuer... " " Je suis dégoûté de la nouvelleté, quelque visage qu'elle porte, et ai raison, car j'en ai vu des effets très dommageables... Ceux qui donnent le branle à un état sont volontiers les premiers absorbés en sa ruine. Le fruit du trouble ne demeure guère à celui qui l'a ému; il bat et brouille l'eau pour d'autres pécheurs. »

L'édition de Port-Royal, XXV, § 6, ajoute la phrase : «mais par un défaut contraire les hommes croient quelquefois pouvoir faire avec justice tout ce qui n'est pas sans exemple.»

4 Sur les révoltes du peuple, voir PINTARD René, Le libertinage érudit..., p. 555. Sorbière, Second discours : «tantôt la sujétion aux puissances souveraines nous abat le courage, tantôt les pensées de liberté nous le relèvent, et nous font insulter témérairement contre les premiers que nous rencontrons en nous relevant. » L'exemple de la Pologne et de la Fronde : "ni libres, ni soumis », les peuples affrontent la souveraineté, qui, pour les vaincre, les dépouille. Une fausse idée d'indépendance soulève contre un ministre bienfaisant une tourbe de naîfs et les réduits à un « état extravagant ».

5 Sur la conduite du peuple et des masses selon les libertins, voir PINTARD, Le libertinage érudit..., p. 555. Naudé, Jugement de tout ce qui a été imprimé contre le cardinal Mazarin, 
nouv. éd. slnd, 1650, p. 668-669 : changeantes, instables, crédules et dangereuses. Il n'approuve pas qu'une Gazette leur donne l'illusion de comprendre quelque chose aux affaires de l'État.

C'est pourquoi le plus sage des législateurs disait que pour le bien des hommes, il faut souvent les piper, et un autre, bon politique, Cum veritatem qua liberetur ignoret, expedit quod fallatur...

Laf. 744, Sel. 618. «Lorsqu'on ne sait pas la vérité d'une chose il est bon qu'il y ait une erreur commune qui fixe l'esprit des hommes comme par exemple la lune à qui on attribue le changement des saisons, le progrès des maladies, etc., car la maladie principale de l'homme est la curiosité inquiète des choses qu'il ne peut savoir et il ne lui est pas si mauvais d'être dans l'erreur que dans cette curiosité inutile. »

Le plus sage des législateurs : renvoi à MONTAIGNE, Essais, II, 12 ; voir CROQUETTE, Pascal et Montaigne, p. 18. Il s'agit de Platon, qui « dit tout détroussément en sa République, que pour le profit des hommes, il est souvent besoin de les piper. » Voir Pensées, éd. Havet, I, Delagrave, 1866, p. 47 : Pascal traite Platon moins favorablement ailleurs.

Laf. 533. "On ne s'imagine Platon et Aristote qu'avec de grandes robes de pédants. C'étaient des gens honnêtes et comme les autres, riant avec leurs amis. Et quand ils se sont divertis à faire leurs lois et leurs politiques ils l'ont fait en se jouant. C'était la partie la moins philosophe et la moins sérieuse de leur vie; la plus philosophe était de vivre simplement et tranquillement. S'ils ont écrit de politique c'était comme pour régler un hôpital de fous. Et s'ils ont fait semblant d'en parler comme d'une grande chose c'est qu'ils savaient que les fous à qui ils parlaient pensaient être rois et empereurs. Ils entrent dans leurs principes pour modérer leur folie au moins mal qu'il se peut. »

Dans Laf. 612, Sel. 505, Pascal dit que Platon dispose au christianisme.

Un autre, bon politique : sans doute Varron.

Il faut la faire regarder comme authentique, éternelle et en cacher le commencement, si on ne veut qu'elle ne prenne bientôt fin : premier jet : et il faut la faire regarder comme authentique éternelle et sans commencement, si on veut qu'elle ne prenne bientôt fin.

Piper : attraper à la glu. Voir JUNGo Michel, Le vocabulaire de Pascal dans les fragments pour une apologie, p. 66.

Cum veritatem qua liberetur ignoret, expedit quod fallatur: "Quand il s'enquiert de cette vérité qui doit le délivrer, on estime qu'il lui est bon d'être pipé. » Saint Augustin, Cité de Dieu, IV, 27, cité par Montaigne, II, 12. Diatribe de saint Augustin contre l'antique religion romaine et ses dignitaires, qui connaissent l'inanité des divinités païennes, mais se refusent à la révéler au peuple.

Pascal remplace le inquirat de Montaigne par ignoret. Il tire ce verbe de la phrase précédente de Montaigne, qui est elle-même une traduction de saint Augustin, $D e$ civitate Dei, IV, 31. Sur Varron : « Ego ista conicere putari debui, nisi evidenter alio loco ipse diceret de religionibus loquens multa esse vera, quae non modo vulgo scire non sit utile, sed etiam, tametsi falsa sunt, aliter existimare populum expediat, et ideo Graecos teletas ac mysteria taciturnitate parietibusque clausisse. » Voir Biblioth. August., t. 33, p. 629. Sur Varron, voir la note p. 813.

MONTAIGNE, Essais, II, 12, éd. Pléiade, p.516. Citation approximative. Le passage de Montaigne touche l'utilisation de la religion par les politiques. Il suit en cela saint 
Augustin. Traduction en marge de l'édition de 1652 : «puisqu'il cherche une vérité qui étant connue lui donnerait la clé des champs, il faut croire que ce qui l'abuse lui est propre. » Sur la déformation apportée par Montaigne à la citation de saint Augustin : celui-ci dit «praeclara religio, quo confugiat liberandus infirmus, et quum veritatem.... » Selon Havet, Pensées, éd. 1866, I, p. 39, n. 2, «belle religion, pour qu'un malade aille y chercher son salut, et que tandis qu'il demande une vérité qui le guérisse, on professe qu'il lui est avantageux d'être trompé.» La déformation qu'effectue Montaigne en ne citant qu'une partie de la phrase apparaît dans la traduction de Villey : « comme il ne cherche la vérité que pour s'affranchir, soyons certains, qu'il est de son intérêt d'être trompé. »

CROQUETTE Bernard, Pascal et Montaigne, p. 129 sq. Pascal supprime la souplesse, les détours et les surprises du texte de Montaigne, II, 12 ; voir la comparaison, p. 20-21. Pascal obtient condensation et systématisation.

Voir PINTARD René, Le libertinage érudit..., p. 548. Sur la doctrine de Gabriel Naudé, Considérations politiques sur les coups d'état: le prince doit mentir à son peuple, «le manier et persuader par belles paroles, le séduire et tromper par les apparences, le gagner et tourner à ses desseins par des prédicateurs et miracles sous prétexte de sainteté ». L'horreur du désordre conduit Naudé à recommander les croyances les plus anciennes, à poursuivre les innovations, à maintenir des légendes dont, en privé, il s'amuse à reconnaître la fausseté.

\section{Remarques sur la partie barrée du folio 70}

COUSIN Victor, Rapport à l'Académie, in CEuvres de M. Victor Cousin, Quatrième série, Littérature, tome I, Paris, Pagnerre, 1849, p. 246 sq.

[13] Mais peut-être que ce sujet passe la portée de la raison. Examinons donc ses inventions...

Quel sujet? On ne peut pas commencer par ce texte. C'est peut-être une accroche à la Martineau.

Les choses de sa force: premier jet, de sa portée. Strowski lit de son parti.

L'un dit que le souverain bien est en la vertu, l'autre le met en la volupté, l'autre à suivre la nature, l'autre en la vérité felix qui potuit rerum cognoscere causas l'autre à l'ignorance tranquille, l'autre en l'indolence, d'autres à résister aux apparences, l'autre à n'admirer rien - nihil mirari prope res una quae possit facere et servare beatum, et les braves pyrrhoniens en leur ataraxie, doute et suspension perpétuelle. Et d'autres plus sages qu'on ne le peut trouver, non pas même par souhait. Nous voilà bien payés.

Texte fourni complet par les Copies. 
En la volupté : Les épicuriens.

L'autre à suivre la nature: DU VAIR ", Philosophie morale des stoïques, éd. Michaux, p. 64. «Pour définir proprement le bien, on peut dire que ce n'est autre chose sinon l'être et l'agir selon la nature. » Sequi naturam.

Ignorance tranquille: Lafuma lit totale. Lafuma suit Tourneur, qui donne aussi totale. Incuriosité, comme dit Montaigne.

Indolence: Absence de souffrance, et non au sens moderne. Idéal stoïcien. Voir Montaigne, Essais, II, 12, 493.

D'autres à résister aux apparences: Formule platonicienne? Sceptique? Stoïcienne? Descartes?

\section{Nihil mirari prope res una quae possit facere et servare beatum}

Les Copies transcrivent admirari.

Horace, Épitres, I, 6, 1.

Aristote doit que la magnanimité consiste à ne rien admirer. Chercher la référence.

Voir Laf. 408, Sel. 27, qui donne admirari.

\section{Les br pyrrhoniens}

On lit parfois braves, parfois vrais.

Sens de brave ? On connaît le sens d'élégant. Il y a aussi le sens de fier-à-bras.

S'agit-il des académistes écoliers?

\section{Ataraxie}

Pascal écrit atiraxie. $\mathrm{C}_{1}$ lit atyranie, $\mathrm{C}_{2}$ tyrannie.

LALANDE André, Vocabulaire..., p. 88. Le mot vient de Démocrite. Employé dans le même sens par les Epicuriens, et par les Stoïciens au sens d'apathie. Elle résulte de la mesure dans le plaisir, de l'harmonie dans la vie.

Pascal l'attribue aux pyrrhoniens, sans doute à partir de Montaigne; voir CROQUETTE, Pascal et Montaigne, p. 20.

MONTAIGNE, Essais, II, 12, éd. Pléiade, p. 483 : « Condition de vie paisible, rassise, exempte des agitations que nous recevons par l'impression de l'opinion et science que nous pensons avoir des choses.» Voir p.650, Pléiade, p. 562 sq., sur l'immobilité du jugement, mais non d'une façon affirmative.

\section{Et d'autres plus sages qu'on ne le peut trouver, non pas même par souhait.}

Qu'on ne le peut : coupé. On doit lire selon les copies.

Non pas même par souhait : me par souhait est coupé.

Brunschvicg transcrit pense trouver un peu mieux.

Comprendre : Et d'autres, qui sont plus sages, soutiennent qu'on ne peut pas le trouver, et qu'on ne peut même pas le souhaiter.

Et par souhait, voir Montaigne, Essais, II, 12, p. 567.

\section{Transposer après les lois article suivant}

Mention placée en marge à gauche. Une accolade réunit les parties comprises entre Il faut voir si... et la fin du folio.

Ce n'est pas un trait qui sépare après les lois et article suivant, mais une pliure. 
Cela concerne spécifiquement ce qui se trouve dans l'accolade : Si faut-il voir si cette belle philosophie...

Voir l'accolade de Rabbinage, où les textes sont très bien centrés par rapport à l'accolade.

Les deux copies n'ont pas transcrit ces mots.

Si faut-il voir si cette belle philosophie n'a rien acquis de certain par un travail si long et si tendu, peut-être qu'au moins l'âme se connaîtra soi-même. Ecoutons les régents du monde sur ce sujet.

Qu'ont-ils pensé de sa substance?

$\mathrm{Si}$ : au sens de encore. A la manière de Montaigne. Voir Essais, II, 12, p. 500.

Tendu : auquel on a consacré tous ses efforts.

Peut-être qu'au moins l'âme se connaitra soi-même: idée reprise dans Disproportion de l'homme.

Régents du monde $: \mathrm{C}_{1}$ lit la substance.

395

Référence de Montaigne, 1652, non reproduite dans les Copies, tout comme 399 plus bas.

\section{Ont-ils été plus heureux à la loger ?}

Double question : où est l'âme dans le temps de la vie? Mais aussi où se logent les âmes séparées des corps?

Qu'ont-ils trouvé de son origine, de sa durée et de son départ?

Espace blanc laissé pour loger la citation, comme dans les Ecrits sur la grâce.

Qu'ont-ils cru, changé en trouvé.

Le mot barré avant cru est demeuré illisible.

Remarques sur les parties barrées du folio 366

Le Transposer après les lois... du folio 70 renvoie probablement à ce passage, qui se trouve en effet après les lois. Cela a donc été écrit après le texte non barré.

Le 13 sert de référence.

Est-ce donc que l'âme est encore un sujet trop noble pour ses faibles lumières? Abaissons-la donc à la matière. Voyons si elle sait de quoi est fait le propre corps qu'elle anime et les autres qu'elle contemple et qu'elle remue à son gré.

Encore n'est pas dans les copies.

Noble : trop élevé. Idée de hiérarchie des sujets : l'âme est supposée plus digne et élevée que le corps. S'oppose à abaisser.

La est une addition. Le mot pose un problème de référent. Le qu'elle anime de la phrase suivante indique que c'est l'âme.

24 De quoi est fait son propre corps semble être la première version. Pascal a tourné court, et mis le propre corps. L'âme n'est pas propriétaire du corps.

Propre : compréhensible du point de vue stylistique et logique ; mais pose un problème philosophique.

Corps est écrit de manière originale, toutes lettres séparées les unes des autres. 


\section{Laf. 61, Sel. 95}

Justice.

Comme la mode fait l'agrément aussi fait-elle la justice.

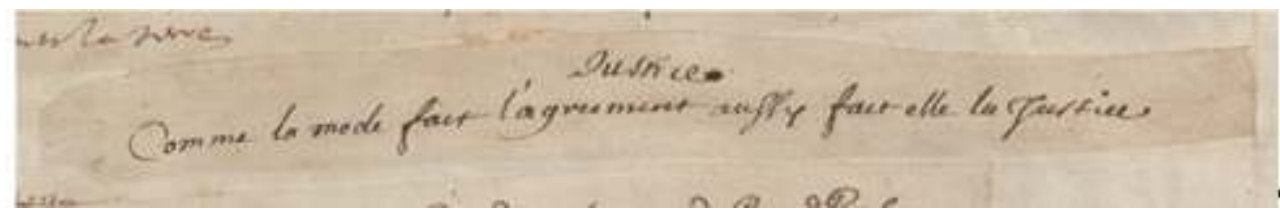

Justice.

Comme la mode fait l'agreement aussy fait elle la Justice.

Le trou d'enfilage est visible.

Fragment hétérographe, y compris le titre, et sans faute d'orthographe. Il semble que ce soit l'écriture du «copiste à l'écriture élégante», comme dans tous les fragments 
non autographes sur papier de type cor couronné sur P / H. Tourneur suppose que c'est l'écriture de Jacqueline Pascal, mais ce n'est pas le cas.

ERNST Pol, Les Pensées de Pascal, Géologie et stratigraphie, p. 294, Annexe 23. 2. E cor sur PH. Sans filigrane.

Le fragment n'est pas dans l'édition de Port-Royal; il se trouve dans le manuscrit de Sainte-Beuve (P') ; il est publié pour la première fois par Condorcet.

Laf. 585, Sel. 486 dit qu'il y a un modèle de l'agrément : cela semble bien contredire ce fragment.

\section{Mode}

Mode : idée de modernité, de goût du moment. Par opposition à ce qui date, à coutume.

Laf. 644, Sel. 529. Peut-ce être autre chose que la complaisance du monde qui vous fasse trouver les choses probables? Nous ferez-vous accroire que ce soit la vérité et que si la mode du duel n'était point, vous trouveriez probable qu'on se peut battre en regardant la chose en elle-même.

Ce fragment est directement lié au sujet, et marque une continuité avec les Provinciales. La mode du duel : cela met en rapport justice et mode.

Laf. 33, Sel. 67. : «Ce qui m’étonne le plus est de voir que tout le monde n'est pas étonné de sa faiblesse. On agit sérieusement et chacun suit sa condition, non pas parce qu'il est bon en effet de la suivre, puisque la mode en est, mais comme si chacun savait certainement où est la raison et la justice. On se trouve déçu à toute heure et par une plaisante humilité on croit que c'est sa faute et non pas celle de l'art qu'on se vante toujours d'avoir. (..).»

Discours sur les passions de l'amour, OC IV, p. 1628, qui indique que « la mode même et les pays règlent souvent ce que l'on appelle beauté. C'est une chose étrange que la coutume se mêle si fort de nos passions. »

Voir la note de Brunschvicg 309. GEF XIII, p. 231.

Laf. 60, Sel. 94. «On la verrait plantée par tous les états du monde, et dans tous les temps, au lieu qu'on ne voit rien de juste ou d'injuste qui ne change de qualité en changeant de climat, trois degrés d'élévation du pôle renversent toute la jurisprudence, un méridien décide de la vérité. En peu d'années de possession les lois fondamentales changent, le droit a ses époques, l'entrée de Saturne au Lion nous marque l'origine d'un tel crime. Plaisante justice qu'une rivière borne. Vérité au-deçà des Pyrénées, erreur au-delà. »

\section{Art d'agréer et de plaire}

Voir L'art de persuader.

MESNARD Jean, Pascal et les Roannez, I, p. 375-376. Méré sur l'art de plaire.

MÉRÉ, Conversations, III, p. 42-43. Différence entre agrément et bon air.

Qu'est-ce que l'agrément? Voir MÉRÉ, Discours, Des agréments, p. 12 sq., qui parle de la gentillesse. 


\section{Laf. 62, Sel. 96}

(Trois hostes)

Qui aurait eu l'amitié du roi d'Angleterre, du roi de Pologne et de la reine de Suède, aurait-il cru manquer de retraite et d'asile au monde?

RO 73-8

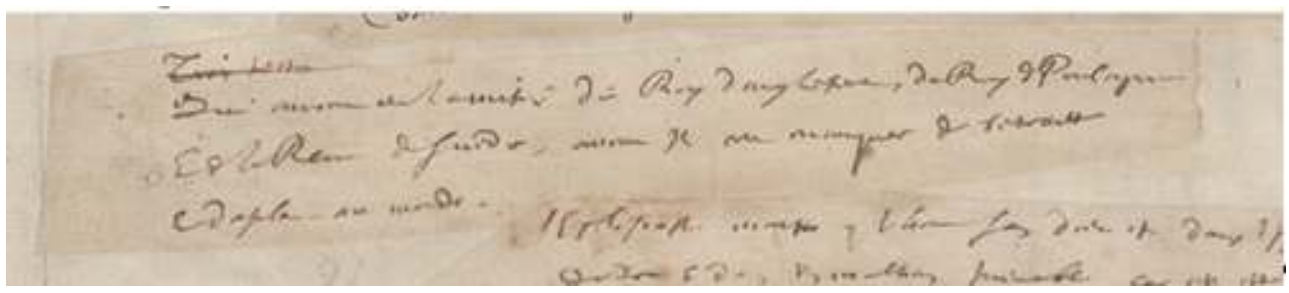

Trois hostes

Qui aurai eul amitié du Roy d angleterre, du Roy de Poulogne

E delaReine deSuede, aurai II cru manquer de retraitte

E d asile - au monde

Le trou d'enfilage est visible.

RO 73-8

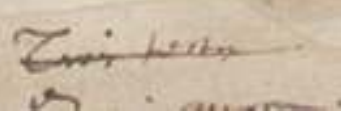

On peut lire Trois testes. Les Copies ne donnent pas les parties barrées, et ne fournissent donc aucune indication.

Pologne : Tourneur lit Pouloigne.

ERNST Pol, Les Pensées de Pascal. Géologie et stratigraphie, p. 166 sq. et p. 173 sq. filigrane cor couronné/ PH complet.

ERNST Pol, Album II, p. 116.

- Laf. 64, Sel. 98, Misère

- Laf. 63, Sel. 97, Misère

- Laf. 62, Sel. 96, Misère

- Laf. 65, Sel. 99, Misère

Restitution très hypothétique, puisque les papiers ne se touchent pas les uns les autres. Les seuls points communs sont les suivants : ces papiers sont tous des bords droits; l'écriture et les encres sont identiques ; la longueur des lignes est identique. 


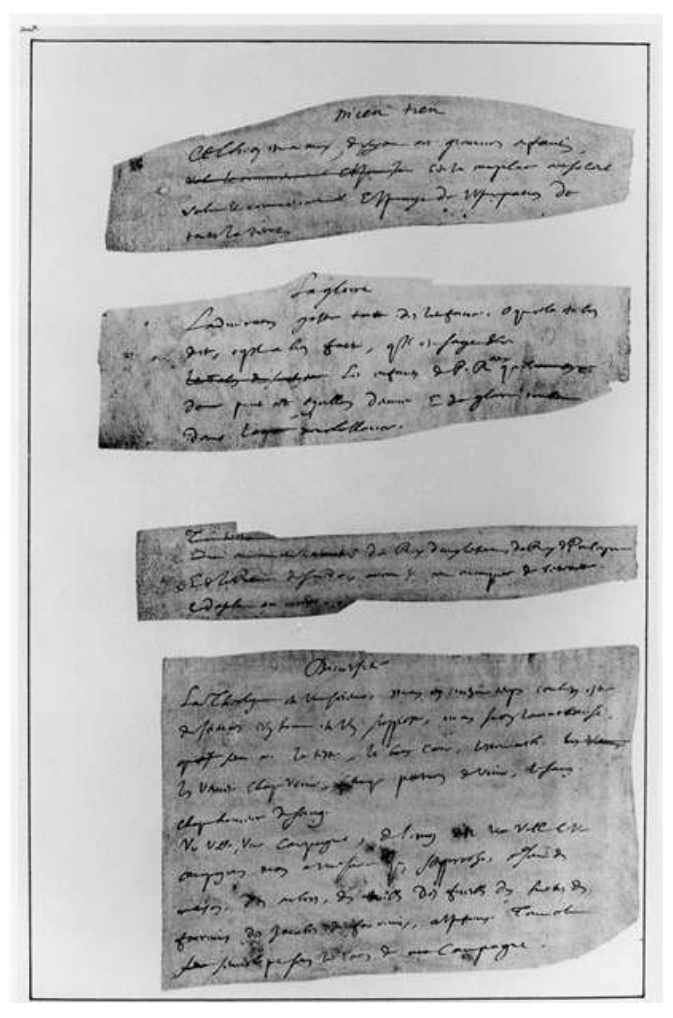

Cliché Pol Ernst

Port-Royal, XXIX, §44, donne le texte comme suit: «Qui aurait eu l'amitié du roi d'Angleterre, du roi de Pologne et de la reine de Suède, aurait-il cru pouvoir manquer de retraite et d'asile au monde? »

Problème: en quoi ceci témoigne-t-il de misère? On peut relier ce thème aux fragments qui marquent la misère des rois qui perdent leur condition. Voir Laf. 116, dans Grandeur, "Toutes ces misères-là même prouvent sa grandeur. Ce sont misères de grand seigneur. Misères d'un roi dépossédé.» La pointe peut porter aussi sur les souverains, pour souligner à quel point il est fréquent qu'ils changent de condition. Les trois souverains mentionnés par Pascal représentent les trois voies par lesquelles les princes quittent leur condition : soit par démission comme Christine de Suède, soit par révolte du peuple comme le roi Jacques d'Angleterre, soit par la guerre comme le roi de Pologne.

Mais à bien examiner le fragment, il n'est pas question ici de la misère du souverain, car si l'on peut considérer Jacques II et Jean-Casimir comme misérables en un sens, en quoi la démission de Christine de Suède peut-elle être considérée comme une marque de misère ? Christine a décidé librement quitter son trône, et sa démission ne manifeste aucune impuissance. Les choses sont prises du point de vue du fuyard sans asile, qui se découvre misérable, faute de protection humaine. Ce qui est en cause, c'est le malheur de celui qui, se croyant assuré de puissants secours dans le monde, se retrouve sans appui, ce qui ne suppose pas nécessairement que les souverains qui le protégeaient aient perdu leur trône, car il peut s'agir tout simplement d'une perte de faveur.

Ernst semble jouer sur le sens du mot hôte, qui peut aussi bien désigner celui qui reçoit que celui qui est reçu. Dans le cas présent, c'est dans le premier sens qu'il doit y avoir 
trois hôtes, qui sont les trois souverains; or les trois que mentionne Ernst sont trois victimes qui ne peuvent être hôtes qu'au second sens.

Il ne rend pas compte du fait que, selon le texte, c'est la possibilité d'avoir trois hôtes, et non pas un seul, qui justifie la réflexion de Pascal. Du reste, le seul indice qui justifie le rapprochement est la mention du roi d'Angleterre, mais Ernst n'arrive à rattacher la reine de Suède et le roi de Pologne que par un artifice.

La fragilité de l'explication rejaillit évidemment sur la datation du fragment, qui n'est pas mieux établie que par les hypothèses précédentes.

Sans doute faut-il prendre le conditionnel initial au sens strict, c'est-à-dire comme une pure hypothèse : Pascal imagine un homme fictif qui aurait connu les trois souverains, et qui se serait retrouvé un jour avec deux fuyards et une démissionnaire pour seuls appuis. Cela dispense de la recherche des clés.

Voir aussi le Psaume II.

LA Rochefoucault, Réflexions diverses, XIX, Des événements de ce siècle, in Maximes, éd. Truchet, Garnier, Pochothèque, 2001, p. 752.

\section{Jean-Casimir, roi de Pologne}

Havet indique que Jean-Casimir, dépossédé de son royaume par les victoires de CharlesGustave, roi de Suède, en 1656, y rentra la même année : c'est donc en 1656 que ce fragment a été écrit.

ERNST Pol, Les Pensées de Pascal. Géologie et stratigraphie, p. 166 sq. Les Nouvelles ordinaires du 29 juillet 1656 annoncent à Paris que « les Polonais avaient pris d'assaut la ville de Varsovie et le château par composition ». La livraison du 5 août 1656 précise que "la ville de Varsovie n'a pas été prise d'assaut comme on avait cru au début, mais par une composition (...); le général Wittemberg (maréchal de camp des armées du roi de Suède) ayant seulement promis de ne pas servir le roi de Suède contre les Polonais ». L' Extraordinaire du 10 août confirme la composition : il est intitulé «Les articles de la reddition de Varsovie». L'article IX précise que ce n'est qu'une trêve de quatre semaines à compter du jour de la présente capitulation. Dans les Nouvelles extraordinaires du 12 août, on apprend que «le roi de Suède n'attend que la venue de l'Électeur de Brandebourg et l'écoulement des eaux qui se sont débordées (...) pour attaquer les Polonais ». Le 2 septembre, les Nouvelles ordinaires font un récit sommaire de «la reprise de Varsovie par le roi de Suède", qui annonce l'Extraordinaire du 6 septembre, intitulé «Le grand combat entre les Suédois et les Polonais près de Varsovie ». On y apprend que le roi de Suède et l'Électeur de Brandebourg ont passé les rivières de Buck et de la Vistule le 27 juillet. Les vainqueurs ont fait leur entrée solennelle dans Varsovie le 31 et le roi de Pologne a quitté la ville dès le $29: p .167$. La défaite de Jean-Casimir est connue vers début septembre 1656 à Paris. Selon Ernst, le rapport avec le fragment Laf. 62, Sel. 96, n'est pas établi, mais il remarque que le terminus a quo de la rédaction serait alors le 2 septembre 1656. Autres chroniques de la Gazette auxquelles on peut rapporter Laf. 62 : p. 167-168.

Le Guern note qu'il est possible que la rédaction date de 1657 : c'est le 17 juin 1657 que les Suédois ont repris Varsovie ; la nouvelle ne fut connue à Paris que dans la seconde moitié du mois de juillet. 


\section{Sur Charles II d'Angleterre}

\section{BIBLIOGRAPHIE}

\section{Bibliographie sommaire sur le droit et la politique chez Pascal}

NOUËT Jacques, Impostures..., V, in Réponses aux lettres provinciales, Liège, Hovius, 1658, p. 111 sq. Pascal choque les « lois civiles à l'étourdi » et « appelle une décision fantasque ce qu'elles font passer pour une maxime inviolable ». Extravagance de savant ridicule et résolu comme Bartole : p. 112. Il ne sait pas les premiers « éléments de la jurisprudence».

ADORNO Francesco Paolo, Pascal, Les Belles Lettres, Paris, 2000, 144 p.

ADORNO Francesco Paolo, « Pascal et le droit naturel », in CLÉRO Jean-Pierre (éd.), Les Pascal à

Rouen, 1640-1648, Colloque de l'Université de Rouen, p. 357-374.

AUERBACH Erich, « Uber Pascal politische theorie », in Vier untersuchungen zur geschishte des französischen bildung, Franeke, Berne, 1951, p. 51-74.

BRIMO A., Pascal et le droit. Essai sur la pensée pascalienne, le problème juridique et les grandes théories du droit et de l'Etat, Sirey, Paris, 1942, 280 p. 
BOUCHILLOUX Hélène, « Justice, force : les limites de la raison d'État selon Pascal », in ZARKA Y. C., Raison et déraison d'État, P.U.F., Paris, 1994, p. 341-357.

CARLO François, «L'idée de société chez Pascal », Revue des Sciences humaines, janv.-mars 1965.

CARRAUD Vincent, « La généalogie de la politique. Pascal », Communio, t.IX, n 3, mai-juin 1984, p. 26-37.

CHINARD Gilbert, En lisant Pascal. Notes sur les Pensées et l'économie du monde, Giard et Droz, Lille et Genève, 1948, 136 p.

CLEMENT Olivier, « Pascal et la politique », Libertés Françaises, 1955, p. 42-66 et p. 107-131.

COUMET Ernest, « La Théorie du Hasard est-elle née par hasard ? ", Annales, mai-juin 1970, p. 574-598.

DEMAHIS Etiennette, La Pensée politique de Pascal, Clermont-Ferrand, 1931.

DEMOREST J.J., « Pascal, les marxistes et l'histoire », L’Esprit créateur, summer 1962, p. 66-73.

DENIS Jacques, « Vues politiques et sociales de Pascal », Mémoires de l'Académie des Sciences, Arts et Belles-Lettres de Caen, 1893, p. 184-216.

DESCOTES Dominique, «La responsabilité collective dans les Provinciales », in DUCHESNE Roger, L'Imposture littéraire dans les Provinciales de Pascal, Publ. Univ. de Provence, Aix, 1985 (2 édition), p. 350-373.

DROULERS Charles, La Cité de Pascal, Paris, 1928.

FERREYROLLES Gérard, « Pascal : politique de la fantaisie », FORCE Pierre et MORGAN David (dir.), De la morale à l'économie politique. Dialogue franco-américain sur les moralistes français. Actes du colloque de Columbia University (New-York), 14-16 octobre 1994, Op. cit., n 6, Université de Pau, 1996, p. 167-174.

FERREYROLLES Gérard, «La politique dans les Pensées », L'Accès aux Pensées de Pascal, Klincksieck, Paris, p. 93-112.

FERREYROLLES Gérard (dir.), Justice et force. Politiques au temps de Pascal, Actes du colloque de Clermont-Ferrand, 20-23 septembre 1990, Klincksieck, Paris, 1996, 384 p.

FERREYROLLES Gérard, Pascal et la raison du politique, Presses Universitaires de France, Paris, 1984.

FERREYROLLES Gérard, Les reines du monde. L'imagination et la coutume chez Pascal, Champion, Paris, 1995, $316 \mathrm{p}$.

FORCE Pierre, « Pascal et Machiavel », FERREYROLLES Gérard (dir.), Justice et force. Politiques au temps de Pascal, Actes du colloque de Clermont-Ferrand, 20-23 septembre 1990, Klincksieck, Paris, 1996, p. 61-70.

FORCE Pierre et MORGAN David (dir.), De la morale à l'économie politique. Dialogue franco-américain sur les moralistes français. Actes du colloque de Columbia University (New-York), 14-16 octobre 1994, Op. cit., nº 6, Université de Pau, 1996.

FUMAROLI Marc, « Pascal et la tradition rhétorique gallicane », Méthodes chez Pascal, p. 359-370.

GLUCK Samuel E., « Pascal, Hobbes and the metaphysics of anarchy », Philosophical quarterly, t. XXXII, 1959-1960, p. 117-125.

GOYET Thérèse, « Le propre de la puissance est de protéger », FERREYROLLES Gérard (dir.), Justice et force. Politiques au temps de Pascal, Actes du colloque de Clermont-Ferrand, 20-23 septembre 1990, Klincksieck, Paris, 1996, p. 333-346. 
JEHASSE Jean et McKENNA Antony, Religion et politique. Les avatars de l'augustinisme, Publications de l'Université de Saint-Étienne, 1998, 412 p.

JUBIN Monique, «L'itinéraire politique dans les Pensées de Pascal », L'Accès aux Pensées de Pascal, Klincksieck, Paris, p. 251-254.

KAWAMATA Koji, « Les soulèvements populaires et Pascal », FERREYROLLES Gérard (dir.), Justice et force. Politiques au temps de Pascal, Actes du colloque de Clermont-Ferrand, 20-23 septembre 1990, Klincksieck, Paris, 1996, p. 237-244.

LAZZERI Christian, Force et justice dans la politique de Pascal, P.U.F., Paris, 1993, 360 p.

MARIN Louis, Le Portrait du Roi, Minuit, Paris, 1981, 304 p.

MARIN Louis, Pascal et Port-Royal, Presses Universitaires de France, Paris, 1997, 424 p.

MARITAIN Jacques, « La politique de Pascal », Revue universelle, t. XIV, n 9, $1^{\mathrm{er}}$ août 1923.

MASPETIOL Roland, « Pascal et le pouvoir », Revue des deux Mondes, № 17, Sept.-oct. 1962, p. 161-174.

McKENNA Antony, « Pascal et Hobbes : les opinions du peuple », FERREYROLLES Gérard (dir.), Justice et force. Politiques au temps de Pascal, Actes du colloque de Clermont-Ferrand, 20-23 septembre 1990, Klincksieck, Paris, 1996, p. 13-26.

McKENNA Antony, «La politique libertine à la lumière des Pensées de Pascal », Littératures classiques, 55, été 2005, p. 77-91.

McKENNA Antony, Entre Descartes et Gassendi. La première édition des Pensées de Pascal, Paris et Oxford, Voltaire Foundation, 1993, p. 154 sq. La pensée politique et l'édition de 1670.

MÉCHOULAN Eric, « Le libertinage politique de Pascal », Littératures classiques, 55, été 2005, p. 93-103.

MESNARD Jean, « Pascal et la Contestation », Revue d'Auvergne, 1971, 3, p. 185-197.

MESNARD Jean, « La monarchie de droit divin, concept anticlérical », FERREYROLLES Gérard (dir.), Justice et force. Politiques au temps de Pascal, Actes du colloque de Clermont-Ferrand, 20-23 septembre 1990, Klincksieck, Paris, 1996, p. 111-138.

MESNARD Jean, « Pascal et la justice à Port-Royal », Commentaire, 121, printemps 2008, p. 163-173.

MESNARD Pierre, L'essor de la philosophie politique au XVI' siècle, Vrin, Paris, 1969.

MOROT-SIR Edouard, « La justice de Dieu selon Pascal », in FERREYROLLES Gérard (dir.), Justice et force. Politiques au temps de Pascal, Actes du colloque de Clermont-Ferrand, 20-23 septembre 1990, Klincksieck, Paris, 1996, p. 281-296.

PÉCHARMAN Martine, « Loi et probabilité. Le problème du droit dans la pensée d'Arnauld, Nicole et Pascal », FERREYROLLES Gérard (dir.), Justice et force. Politiques au temps de Pascal, Actes du colloque de Clermont-Ferrand, 20-23 septembre 1990, Klincksieck, Paris, 1996, p. 161-186.

PÉCHARMAN Martine, « Pascal et le politique », in CORNETTE J. et MECHOULAN H. (dir.), L'Etat classique, Vrin, 1996, p. 113-132.

PÉCHARMAN Martine (dir.), Les « trois ordres » de Pascal, Revue de Métaphysique et de Morale, $\mathrm{n}^{\circ} 1$, mars 1997, Presses Universitaires de France, Paris, 1997.

PERES André, « L'État chez Montaigne, La Boétie, Pascal », FERREYROLLES Gérard (dir.), Justice et force. Politiques au temps de Pascal, Actes du colloque de Clermont-Ferrand, 20-23 septembre 1990, Klincksieck, Paris, 1996, p. 71-82. 
PLAINEMAISON Jacques, « Pascal et la "politique" des Jésuites. Étude de la forme "politique" dans les Provinciales », FERREYROLLES Gérard (dir.), Justice et force. Politiques au temps de Pascal, Actes du colloque de Clermont-Ferrand, 20-23 septembre 1990, Klincksieck, Paris, 1996, p. 255-266.

PUCELLE Jean, « La Politique de Pascal et la doctrine des trois ordres », Chroniques de Port-Royal, $\mathrm{n}^{\circ} 10,1959$, p. 8-21.

REGUIG-NAYA Delphine, Le corps des idées : pensées et poétiques du langage dans l'augustinisme du second Port-Royal, p. 319.

ROHOU Jean, « Pour un ordre social fondé sur l'intérêt : Pascal, Silhon, Nicole et Domat à l'aube de l'ère libérale », FERREYROLLES Gérard (dir.), Justice et force. Politiques au temps de Pascal, Actes du colloque de Clermont-Ferrand, 20-23 septembre 1990, Klincksieck, Paris, 1996, p. 207-222.

SECRETAN Philibert, « Pensées politiques de Pascal rapportées à la théorie des trois ordres ", Freiburger Zeitschrift für Philosophie und Theologie, H, 1-2.

SELLIER Philippe, Pascal et saint Augustin, Paris, Colin, 1970, p. 197 sq. Politique chez Pascal. La cité mauvaise.

SELLIER Philippe, « De la tyrannie », FERREYROLLES Gérard (dir.), Justice et force. Politiques au temps de Pascal, Actes du colloque de Clermont-Ferrand, 20-23 septembre 1990, Klincksieck, Paris, 1996, p. 365-374.

SHIOKAWA Tetsuya, « La Guerre et la Paix selon Pascal », Pascal, Port-Royal, Orient, Occident, Klincksieck, 1991, p. 319-327.

SIMON Pierre-Henri, « Pascal, l'Histoire et la justice », in Le Jardin et la ville, Le Seuil, Paris, 1962, p. 46-58.

STROWSKI Fortunat, Pascal et son temps, III, Paris, 1908.

TAVENEAUX René, Jansénisme et politique, Colin, Paris, 1965.

THIROUIN Laurent, « Le réalisme de Pascal », FERREYROLLES Gérard (dir.), Justice et force. Politiques au temps de Pascal, Actes du colloque de Clermont-Ferrand, 20-23 septembre 1990, Klincksieck, Paris, 1996, p. 347-364.

ZARKA Yves-Charles, Hobbes et la pensée politique moderne, P.U.F., Paris, 1995, 308 p.

ZARKA Yves Charles, Philosophie et politique à l'âge classique, Presses Universitaires de France, Paris, 1998, 296 p.

ZARKA Yves-Charles, « Les implications politiques des trois ordres selon Pascal », Revue de métaphysique et de morale, janv.-mars 1997, nº 1, p. 97-106.

INDEX

Mots-clés : Pascal, Pensées

\section{AUTEUR}

\section{DOMINIQUE DESCOTES}

CERHAC, Université Blaise Pascal 